TRANSACTIONS OF THE

AMERICAN MATHEMATICAL SOCIETY

Volume 349, Number 7, July 1997, Pages 2699-2719

S 0002-9947(97)01604-8

\title{
ISOMORPHISM OF LATTICES OF RECURSIVELY ENUMERABLE SETS
}

\author{
TODD HAMMOND
}

\begin{abstract}
Let $\omega=\{0,1,2, \ldots\}$, and for $A \subseteq \omega$, let $\mathcal{E}^{A}$ be the lattice of subsets of $\omega$ which are recursively enumerable relative to the "oracle" $A$. Let $\left(\mathcal{E}^{A}\right)^{*}$ be $\mathcal{E}^{A} / \mathcal{I}$, where $\mathcal{I}$ is the ideal of finite subsets of $\omega$. It is established that for any $A, B \subseteq \omega,\left(\mathcal{E}^{A}\right)^{*}$ is effectively isomorphic to $\left(\mathcal{E}^{B}\right)^{*}$ if and only if $A^{\prime} \equiv_{T} B^{\prime}$, where $A^{\prime}$ is the Turing jump of $A$. A consequence is that if $A^{\prime} \equiv_{T} B^{\prime}$, then $\mathcal{E}^{A} \cong \mathcal{E}^{B}$. A second consequence is that $\left(\mathcal{E}^{A}\right)^{*}$ can be effectively embedded into $\left(\mathcal{E}^{B}\right)^{*}$ preserving least and greatest elements if and only if $A^{\prime} \leq_{T} B^{\prime}$
\end{abstract}

\section{INTRODUCTION}

Many theorems in recursion theory remain true when relativized to any oracle $A$ : that is, when "r.e." is replaced throughout by "r.e. in $A$ ", "recursive" is replaced by "recursive in $A$ ", and so on. This is because most of the methods of proof used in recursion theory actually use only the fact that the class of recursive functions is closed under certain operations. Nevertheless, if we define $\mathcal{E}$ to be the lattice of recursively enumerable sets and $\mathcal{E}^{A}$ to be the lattice of sets which are recursively enumerable in $A$, it is not true that for all sentences $\varphi$ (e.g., of second order logic $\left.\mathcal{L}_{I I}\right), \varphi$ is true in $\mathcal{E}$ if and only if $\varphi$ is true in $\mathcal{E}^{A}$. In particular, there are sets $A$ such that $\mathcal{E}^{A} \neq \mathcal{E}$ (see Lachlan [3], Feiner [1], or Hammond [2]). ${ }^{1}$ In this paper, we will establish that there are nonrecursive sets $A$ such that $\mathcal{E}^{A} \cong \mathcal{E}$. Indeed, let $\left(\mathcal{E}^{A}\right)^{*}$ be the lattice $\mathcal{E}^{A}$ modulo the ideal of finite sets; for $U \in \mathcal{E}^{A}$, let $U^{*}$ be the unique element of $\left(\mathcal{E}^{A}\right)^{*}$ containing $U$; let $\omega$ be the set of natural numbers; and say that $\left(\mathcal{E}^{A}\right)^{*}$ and $\left(\mathcal{E}^{B}\right)^{*}$ are effectively isomorphic $\left(\left(\mathcal{E}^{A}\right)^{*} \cong_{1}\left(\mathcal{E}^{B}\right)^{*}\right)$ if and only if there is a recursive permutation $f$ of $\omega$ such that the map $\left(W_{e}^{A}\right)^{*} \mapsto\left(W_{f(e)}^{B}\right)^{*}$ is a well-defined isomorphism of $\left(\mathcal{E}^{A}\right)^{*}$ and $\left(\mathcal{E}^{B}\right)^{*}$. Then we will establish the following more general theorem, which is the main theorem of this paper:

Theorem 1. For all $A, B \subseteq \omega,\left(\mathcal{E}^{A}\right)^{*} \cong_{1}\left(\mathcal{E}^{B}\right)^{*}$ if and only if $A^{\prime} \equiv_{T} B^{\prime}$.

We refer the reader to Soare [9] for notions we leave undefined here: in particular for the definitions of $A^{\prime}$ (the Turing jump of $A$ ), the relations $\leq_{T}$ and $\equiv_{T}$

Received by the editors December 17, 1991 and, in revised form, August 3, 1995.

1991 Mathematics Subject Classification. Primary 03D25.

Key words and phrases. Effective isomorphism, effectively isomorphic, recursively enumerable, oracle, Turing jump, effective embedding, effectively embeddable.

This paper is based primarily on part of the author's Ph.D. thesis, written under the supervision of Professor Robert Vaught.

${ }^{1}$ Thus we can simply let $\varphi$ be such that $\varphi$ is true in a model $\mathcal{M}$ if and only if $\mathcal{M} \cong \mathcal{E}$. The minimum possible complexity of such a $\varphi$ is, however, unknown.

(C)1997 American Mathematical Society 
(Turing reduction and equivalence), $W_{e}^{A}$ (the " $e$-th subset of $\omega$ which is recursively enumerable in $A$ "), and $\{e\}^{A}$ (the " $e$-th function which is partial recursive in $A$ "). Notice that because all acceptable enumerations of the r.e. sets are equivalent up to a recursive permutation of $\omega$ (see Rogers [5]), the definition of $\cong_{1}$ is independent of the choice of an acceptable enumeration of the r.e. sets.

The main goal of this paper is to prove Theorem 1 . Theorem 1 has the following easy corollary:

Corollary 1. For all $A, B \subseteq \omega$, if $A^{\prime} \equiv_{T} B^{\prime}$, then $\mathcal{E}^{A} \cong \mathcal{E}^{B}$.

Proof. Suppose $A^{\prime} \equiv_{T} B^{\prime}$. Thus $\left(\mathcal{E}^{A}\right)^{*} \cong_{1}\left(\mathcal{E}^{B}\right)^{*}$ by the right to left direction of Theorem 1, so a fortiori $\left(\mathcal{E}^{A}\right)^{*} \cong\left(\mathcal{E}^{B}\right)^{*}$. But Lachlan [3] established (Lemma 14, p. 28) that if $\mathcal{M}_{1}$ and $\mathcal{M}_{2}$ are two countable lattices of subsets of $\omega$ which each contain all finite and cofinite sets, then $\mathcal{M}_{1} \cong \mathcal{M}_{2}$ if and only if $\mathcal{M}_{1}^{*} \cong \mathcal{M}_{2}^{*}$. Thus $\mathcal{E}^{A} \cong \mathcal{E}^{B}$.

An immediate and interesting consequence of Corollary 1 is that $\mathcal{E}^{A} \cong \mathcal{E}$ for any set $A \subseteq \omega$ such that $A^{\prime} \equiv_{T} \emptyset^{\prime}$ (i.e., such that $A$ is low). Roughly speaking, this is interesting because, with the use of Corollary 1, it shows that all theorems "about" the lattice $\mathcal{E}$ must relativize to any low oracle: i.e., all theorems "about" $\mathcal{E}$ will remain true if "recursively enumerable" is replaced throughout by "recursively enumerable in $A$ " for some low $A$. The lattice $\mathcal{E}$ is a complex structure, and is far from being completely understood.

There is an analog of Theorem 1 which holds for effective embeddings. Here we say that $\Phi:\left(\mathcal{E}^{A}\right)^{*} \rightarrow\left(\mathcal{E}^{B}\right)^{*}$ is an effective embedding if $\Phi$ is an embedding of $\left(\mathcal{E}^{A}\right)^{*}$ into $\left(\mathcal{E}^{B}\right)^{*}$ and if there is a recursive function $f: \omega \rightarrow \omega$ such that $\Phi\left(\left(W_{e}^{A}\right)^{*}\right)=\left(W_{f(e)}^{B}\right)^{*}$ for all $e$. Then:

Corollary 2. For all $A, B \subseteq \omega,\left(\mathcal{E}^{A}\right)^{*}$ can be effectively embedded into $\left(\mathcal{E}^{B}\right)^{*}$ preserving least and greatest elements if and only if $A^{\prime} \leq_{T} B^{\prime}$.

Proof of $\Leftarrow$. Suppose $A^{\prime} \leq_{T} B^{\prime}$. By the Relativized Friedberg Completeness Criterion (see Soare [9], p. 98), there is a set $C$ such that $A \leq_{T} C$ and $C^{\prime} \equiv_{T} B^{\prime}$. Since $A \leq_{T} C$, the inclusion map from $\left(\mathcal{E}^{A}\right)^{*}$ to $\left(\mathcal{E}^{C}\right)^{*}$ is easily an effective embedding preserving least and greatest elements. But $C^{\prime} \equiv_{T} B^{\prime}$, so by Theorem $1,\left(\mathcal{E}^{C}\right)^{*}$ and $\left(\mathcal{E}^{B}\right)^{*}$ are effectively isomorphic. Composing these maps, we get an effective embedding of $\left(\mathcal{E}^{A}\right)^{*}$ into $\left(\mathcal{E}^{C}\right)^{*}$ preserving least and greatest elements.

The proof of the left to right direction of Corollary 2 will be delayed until Section 2. The following curious "Schröder-Bernstein"-like result follows easily from Theorem 1 and Corollary 2:

Corollary 3. For all $A, B \subseteq \omega,\left(\mathcal{E}^{A}\right)^{*}$ is effectively isomorphic to $\left(\mathcal{E}^{B}\right)^{*}$ if and only if each of $\left(\mathcal{E}^{A}\right)^{*}$ and $\left(\mathcal{E}^{B}\right)^{*}$ can be effectively embedded into the other preserving least and greatest elements.

Proof. By Theorem 1 and Corollary 2, this reduces to saying that $A^{\prime} \equiv_{T} B^{\prime}$ if and only if $A^{\prime} \leq_{T} B^{\prime}$ and $B^{\prime} \leq_{T} A^{\prime}$.

However, it is not hard to see that Corollary 3 would be false if the word "effective" were dropped.

The left to right directions of Theorem 1 and Corollary 2 are easy, and will be proven in Section 2. It will then remain to prove the right to left direction of Theorem 1. We will do this in Sections 3-6 using a modification of Soare's automorphism 
construction [7]. Section 3 gives some background on Soare's automorphism construction, an outline of what is done in Sections 4 through 6 , and briefly explains the most important difference between our proof and proofs of other theorems which also use modifications of Soare's automorphism construction.

Although this paper is in principle self-contained, it will be helpful for the reader to be familiar with the essentials of Soare's automorphism method. Sections XV.6.1 and XV.6.3 of Soare [9], which give some of the intuition and motivation for his automorphism method, will be particularly helpful in this regard. The reader should keep in mind, however, that some features of his construction will be somewhat modified.

We adopt the usual conventions of set theory: in particular, for all $n \in \omega$, $n=\{i \in \omega \mid i<n\}$. If $f$ is a function, then $f=\operatorname{graph} f=\{(x, y) \mid x \in \operatorname{dom} f$ and $f(x)=y\} . P(\omega)=\{A \mid A \subseteq \omega\}$. We will normally use the symbols $A, B$, and $C$ to denote subsets of $\omega$, and the symbols $a$ through $e$ and $i$ through $z$ to denote elements of $\omega$. A function $F$ is a $(1, n)$-ary partial recursive functional if $\operatorname{dom} F \subseteq P(\omega) \times \omega^{n}$ and if there is an $e \in \omega$ such that graph $F=\left\{\left(\left(A, x_{1}, \ldots, x_{n}\right), y\right) \in\left(P(\omega) \times \omega^{n}\right) \times \omega \mid\right.$ $\{e\}^{A}\left(x_{1}, \ldots, x_{n}\right)$ exists and equals $\left.y\right\}$. If $A \subseteq \omega$, we let $F^{A}$ be the function with graph $\left\{\left(\left(x_{1}, \ldots, x_{n}\right), y\right) \in \omega^{n} \times \omega \mid F\left(A, x_{1}, \ldots, x_{n}\right)\right.$ exists and equals $\left.y\right\}$. If $n>0$ and $a \in \omega$ we let $F_{a}$ be the function with graph $\left\{\left(\left(A, x_{2}, \ldots, x_{n}\right), y\right) \in\right.$ $\left(P(\omega) \times \omega^{n-1}\right) \times \omega \mid F\left(A, a, x_{1}, \ldots, x_{n}\right)$ exists and equals $\left.y\right\}$. If $f$ is a function with domain a subset of $\omega$, then we say that $\lim _{s} f(s)$ exists if there exist $s \in \omega$ and $\alpha$ such that for all $t \geq s, t \in \operatorname{dom} f$ and $f(t)=\alpha$, in which case we put $\lim _{s} f(s)=\alpha$.

A few remarks on the context of this paper: The problem of the relationship between $\mathcal{E}$ and the lattices $\mathcal{E}^{A}$ might be called the "outer" isomorphism problem. The "inner" isomorphism problem is then the problem of the relationship between $\mathcal{E}$ and the lattices $\mathcal{L}(A)$, where for $A$ an r.e. set, $\mathcal{L}(A)=\{W \in \mathcal{E} \mid W \supseteq A\}$. Both problems (and especially the "inner" isomorphism problem) are in the spirit of Post, who was among the first to ask questions about the relationship between the complexity of a set and its lattice-theoretic properties. The "inner" isomorphism problem has met with much success in work by Soare [8], Maass [4], and in recent (as yet unpublished) work by Harrington, Lachlan, Maass, and Soare. The "outer" isomorphism problem has until recently been relatively neglected, important exceptions being in work by Lachlan [3] and Feiner [1]. The main theorem of this paper is most closely analogous to the main theorem of Maass [4], which indeed supplied much inspiration for this paper.

\section{Proof of the easy direction of the Main Theorem}

The following proposition is well known (see Soare [9], pp. 53 and 66):

Proposition 1. Let $A, B \subseteq \omega$. Then $B^{\prime} \leq_{T} A^{\prime}$ if and only if there is a recursive $f: \omega \rightarrow \omega$ such that for all $e \in \omega, W_{e}^{B}$ is infinite if and only if $W_{f(e)}^{A}$ is infinite. $A^{\prime} \equiv_{T} B^{\prime}$ if and only if there is a recursive permutation $f$ of $\omega$ such that for all $e \in \omega, W_{e}^{B}$ is infinite if and only if $W_{f(e)}^{A}$ is infinite.

Proposition 1 immediately gives the left to right directions of Theorem 1 and Corollary 2:

Proof of $\Rightarrow$ for Corollary 2. Let $f: \omega \rightarrow \omega$ be a recursive function such that the function $F:\left(\mathcal{E}^{A}\right)^{*} \rightarrow\left(\mathcal{E}^{B}\right)^{*}$ with $F\left(\left(W_{e}^{A}\right)^{*}\right)=\left(W_{f(e)}^{B}\right)^{*}$ for all $e$ is well-defined 
and is an embedding of $\left(\mathcal{E}^{A}\right)^{*}$ into $\left(\mathcal{E}^{B}\right)^{*}$ preserving least and greatest elements. But then $\left(W_{e}^{A}\right)^{*}=\emptyset^{*}$ if and only if $\left(W_{f(e)}^{B}\right)^{*}=\emptyset^{*}$. I.e., $W_{e}^{A}$ is finite if and only if $W_{f(e)}^{B}$ is finite. Hence by Proposition $1, A^{\prime} \leq_{T} B^{\prime}$, as desired.

Proof of $\Rightarrow$ for Theorem 1. Strictly analogous, except we can now choose $f$ to be a recursive permutation. (Alternatively, we can apply the proof above twice: first to get $A^{\prime} \leq_{T} B^{\prime}$, and second to get $B^{\prime} \leq_{T} A^{\prime}$.)

Proposition 1 also plays an important role in the proof of the hard direction of Theorem 1.

\section{INTRODUCTION TO THE HARD DIRECTION}

In Sections 4-6 we will prove the harder direction of Theorem 1. That is, given $A, B \subseteq \omega$ such that $A^{\prime} \equiv_{T} B^{\prime}$, we will prove that $\left(\mathcal{E}^{A}\right)^{*} \cong_{1}\left(\mathcal{E}^{B}\right)^{*}$. Our proof will use the automorphism method (though not the results) of Soare [7] with simplifications taken from Maass [4] and Soare [9]. Of course, other changes will also be necessary. Maass's notion of verified [4] inspired a similar notion which will be an important element of our proof (this notion will be defined in Section 4). However, it is not necessary for the reader to have read these proofs. Throughout the rest of the paper, we fix sets $A, B \subseteq \omega$ such that $A^{\prime} \equiv_{T} B^{\prime}$.

To describe the proof of the hard direction further, we need the following notions, which we will use frequently throughout the rest of the paper:

Definition. (i) $\nu$ is a state if $\nu$ is a triple $(e, \sigma, \tau)$ where $e \in \omega$ and $\sigma$ and $\tau$ are subsets of $\{0,1,2, \ldots, e\}$. If $\nu=(e, \sigma, \tau)$ is a state, then $|\nu|$ (the length of $\nu)$ is $e$, and $\nu^{*}=(e, \tau, \sigma)$. Let $S$ be the set of all states.

(ii) If $\left(X_{i}\right)_{i \in \omega}$ and $\left(Y_{i}\right)_{i \in \omega}$ are sequences of subsets of $\omega$, and if $x, e \in \omega$, then we let $\nu\left(e, x ;\left(X_{i}\right)_{i \in \omega},\left(Y_{i}\right)_{i \in \omega}\right)$ be the state $\left(e,\left\{i \leq e \mid x \in X_{i}\right\},\left\{i \leq e \mid x \in Y_{i}\right\}\right)$.

We will use the symbols $\nu, \mu$, and $\pi$ for states. States can be easily coded by elements of $\omega$, and it is sometimes convenient to identify $S$ with $\omega$.

A sketch of our modification of Soare's automorphism construction is as follows: In Section 5, we will use Smullyan's Double Recursion Theorem and the definitions of Section 4 to define sequences $\left(U_{i}\right)_{i \in \omega}$ and $\left(\hat{V}_{i}\right)_{i \in \omega}$ of recursively-enumerable-in$A$ subsets of $\omega$ and sequences $\left(V_{i}\right)_{i \in \omega}$ and $\left(\hat{U}_{i}\right)_{i \in \omega}$ of recursively-enumerable-in- $B$ subsets of $\omega$. Given $e, x \in \omega$, we will define $\nu(e, x)=\nu\left(e, x ;\left(U_{i}\right)_{i \in \omega},\left(\hat{V}_{i}\right)_{i \in \omega}\right)$ and $\hat{\nu}(e, x)=\nu\left(e, x ;\left(V_{i}\right)_{i \in \omega},\left(\hat{U}_{i}\right)_{i \in \omega}\right)$. By the end of Section 6 , we will see that the sequences $\left(U_{i}\right)_{i \in \omega},\left(\hat{V}_{i}\right)_{i \in \omega},\left(V_{i}\right)_{i \in \omega}$, and $\left(\hat{U}_{i}\right)_{i \in \omega}$ have the following properties:

(1) $U_{i}={ }^{*} W_{i}^{A}$ and $V_{i}={ }^{*} W_{i}^{B}$ for all $i$.

(2) For every state $\mu,\{x \mid \nu(|\mu|, x)=\mu\}$ is infinite $\Leftrightarrow\left\{x \mid \hat{\nu}(|\mu|, x)=\mu^{*}\right\}$ is infinite.

It now follows (as the reader can easily check) that (1), (2), and the facts that $\hat{V}_{i} \in \mathcal{E}^{A}$ and $\hat{U}_{i} \in \mathcal{E}^{B}$ for all $i$ imply that the relation $R=\left\{\left(U_{i}^{*}, \hat{U}_{i}^{*}\right) \mid i \in\right.$ $\omega\} \cup\left\{\left(\hat{V}_{i}^{*}, V_{i}^{*}\right) \mid i \in \omega\right\}$ is a function and is an isomorphism from $\left(\mathcal{E}^{A}\right)^{*}$ to $\left(\mathcal{E}^{B}\right)^{*}$.

Moreover, the sequences $\left(\hat{V}_{i}\right)_{i \in \omega}$ and $\left(\hat{U}_{i}\right)_{i \in \omega}$ will be defined in such a way that there are recursive functions $g, h: \omega \rightarrow \omega$ such that

(3) $\hat{V}_{i}={ }^{*} W_{g(i)}^{A}$ and $\hat{U}_{i}={ }^{*} W_{h(i)}^{B}$ for all $i \in \omega$.

(In fact, (3) will also hold with "=*" replaced by "=".)

It is easy to see (cf. Soare [9], p. 344) that (1)-(3) imply that 
(4) $\left(\mathcal{E}^{A}\right)^{*}$ and $\left(\mathcal{E}^{B}\right)^{*}$ are effectively isomorphic via an isomorphism $\Phi$ such that for all $i, \Phi\left(U_{i}^{*}\right)=\hat{U}_{i}^{*}$ and $\Phi\left(\hat{V}_{i}^{*}\right)=V_{i}^{*}$.

We will define the sequences $\left(U_{i}\right)_{i \in \omega},\left(\hat{V}_{i}\right)_{i \in \omega},\left(V_{i}\right)_{i \in \omega}$, and $\left(\hat{U}_{i}\right)_{i \in \omega}$ by "stages". That is, we will define sequences $\left(U_{i, s}\right)_{i, s \in \omega},\left(\hat{V}_{i, s}\right)_{i, s \in \omega},\left(V_{i, s}\right)_{i, s \in \omega}$, and $\left(\hat{U}_{i, s}\right)_{i, s \in \omega}$ of finite sets, and for all $i \in \omega$ will put $U_{i}=\bigcup_{s} U_{i, s}, \hat{V}_{i}=\bigcup_{s} \hat{V}_{i, s}, V_{i}=\bigcup_{s} V_{i, s}$, and $\hat{U}_{i}=\bigcup_{s} \hat{U}_{i, s}$. Also, for all $i, s \in \omega$, we will have $U_{i, s} \subseteq U_{i, s+1}, \hat{V}_{i, s} \subseteq \hat{V}_{i, s+1}$, $V_{i, s} \subseteq V_{i, s+1}$, and $\hat{U}_{i, s} \subseteq \hat{U}_{i, s+1}$.

At the risk of oversimplifying slightly, it is fair to say that the most important difference between our proof and the proofs of other theorems which also use modifications of Soare's automorphism construction is that the sequences $\left(\hat{V}_{i}\right)_{i \in \omega}$ and $\left(\hat{U}_{i}\right)_{i \in \omega}$, whose definitions have in other modifications of Soare's automorphism construction seemed intertwined in a complex way, are now defined entirely separately except for one use of Smullyan's Double Recursion Theorem. (The statement of Smullyan's Double Recursion Theorem is given in Section 5.) Roughly speaking, the reason that this change is necessary is that in order to achieve property (3), the sequences $\left(\hat{V}_{i}\right)_{i \in \omega}$ and $\left(\hat{U}_{i}\right)_{i \in \omega}$ must be defined using different "oracles" ( $A$ and $B$, respectively). In effect, and now very roughly speaking, in defining $\left(\hat{V}_{i, s}\right)_{i \in \omega}$ at some $s$, we will have no knowledge of $\left(\hat{U}_{i, \hat{s}}\right)_{i \in \omega}$ at any particular $\hat{s}$, but can only guess what will happen in $\left(\hat{U}_{i, \hat{s}}\right)_{i \in \omega}$ "infinitely often" (i.e., for infinitely many $\hat{s}$ ).

We can approximate the final state $\nu(e, x)$ or $\hat{\nu}(e, \hat{x})$ of an element $x$ or $\hat{x}$ of $\omega$ using the following functions: let $\nu_{s}(e, x)=\nu\left(e, x ;\left(U_{i, s}\right)_{i \in \omega},\left(\hat{V}_{i, s}\right)_{i \in \omega}\right)$ and $\hat{\nu}_{s}(e, \hat{x})=\nu\left(e, \hat{x} ;\left(V_{i, s}\right)_{i \in \omega},\left(\hat{U}_{i, s}\right)_{i \in \omega}\right)$. If $\nu=(e, \sigma, \tau)$ and $\nu^{\prime}=\left(e^{\prime}, \sigma^{\prime}, \tau^{\prime}\right)$ are states, then we put $\nu \subseteq \nu^{\prime}$ if and only if $e=e^{\prime}, \sigma \subseteq \sigma^{\prime}$ and $\tau \subseteq \tau^{\prime}$. Thus we see that for all $e, x, \hat{x}, s \in \omega, \nu_{s}(e, x) \subseteq \nu_{s+1}(e, x)$ and $\hat{\nu}_{s}(e, \hat{x}) \subseteq \hat{\nu}_{s+1}(e, \hat{x})$. Now consider the following problem: If we are given $e, x, \hat{x}, s, \hat{s} \in \omega$ and sets $\left(U_{i, s}\right)_{i \in \omega},\left(\hat{V}_{i, s}\right)_{i \in \omega}$, $\left(V_{i, \hat{s}}\right)_{i \in \omega}$, and $\left(\hat{U}_{i, \hat{s}}\right)_{i \in \omega}$, under what conditions on $\nu_{s}(e, x)$ and $\hat{\nu}_{\hat{s}}(e, \hat{x})$ can we define $\left(\hat{V}_{i, s+1}\right)_{i \in \omega}$ and $\left(\hat{U}_{i, \hat{s}+1}\right)_{i \in \omega}$ so that $\nu_{s+1}(e, x)=\hat{\nu}_{\hat{s}+1}(e, \hat{x})$, and yet also let $U_{i, s+1}=U_{i, s}$ and $V_{i, \hat{s}+1}=V_{i, \hat{s}}$ for all $i \in \omega$ ? The answer uses the following definition:

Definition. Let $\nu=(e, \sigma, \hat{\tau})$ and $\hat{\nu}=(\hat{e}, \tau, \hat{\sigma})$ be states. Then we put

(i) $\nu \| \hat{\nu}(\operatorname{read} \nu$ is compatible with $\hat{\nu})$ iff $e=\hat{e}, \hat{\sigma} \subseteq \sigma$ and $\hat{\tau} \subseteq \tau$.

(ii) $\nu_{*} \| \hat{\nu}$ iff $e=\hat{e}, \hat{\sigma} \subseteq \sigma$ and $\hat{\tau}=\tau$.

(iii) $\nu \|_{*} \hat{\nu}$ iff $e=\hat{e}, \hat{\sigma}=\sigma$ and $\hat{\tau} \subseteq \tau$.

Notice that $\|$ is a symmetric relation.

It is easy to see that we can define $\left(\hat{V}_{i, s+1}\right)_{i \in \omega}$ and $\left(\hat{U}_{i, \hat{s}+1}\right)_{i \in \omega}$ as desired if and only if $\nu_{s}(e, x) \| \hat{\nu}_{\hat{s}}(e, \hat{x})$. Moreover, if $\nu_{s}(e, x) * \| \hat{\nu}_{\hat{s}}(e, \hat{x})$, we can in addition take $\hat{V}_{i, s+1}=\hat{V}_{i, s}$ for all $i$, and if $\nu_{s}(e, x) \|_{*} \hat{\nu}_{\hat{s}}(e, \hat{x})$, we can take $\hat{U}_{i, s+1}=\hat{U}_{i, s}$ for all $i$.

Definition. Let $\nu=(e, \sigma, \tau)$ and $\nu^{\prime}=\left(e^{\prime}, \sigma^{\prime}, \tau^{\prime}\right)$ be states. Then we put

(i) $\nu \geq \nu^{\prime}\left(\operatorname{read} \nu\right.$ covers $\left.\nu^{\prime}\right)$ iff $e=e^{\prime}, \sigma \supseteq \sigma^{\prime}$ and $\tau \subseteq \tau^{\prime}$.

(ii) $\nu \geq_{*} \nu^{\prime}$ (read $\nu$ exactly covers $\left.\nu^{\prime}\right)$ iff $e=e^{\prime}, \tau=\tau^{\prime}$ and $\sigma \supseteq \sigma^{\prime}$.

The intuition is that bigger is better: it is easier for $\leq$-big states to be compatible than it is for $\leq$-smaller ones to be, in the sense of the following easy proposition:

Proposition 1. Let $\nu, \nu_{1}, \hat{\nu}, \hat{\nu}_{1}$ be states. Then:

(i) If $\nu \| \hat{\nu}$ and if $\nu_{1} \geq \nu$ and $\hat{\nu}_{1} \geq \hat{\nu}$, then $\nu_{1} \| \hat{\nu}_{1}$.

(ii) If $\nu_{*} \| \hat{\nu}$ and if $\nu_{1} \geq_{*} \nu$, then $\nu_{1 *} \| \hat{\nu}$. 
(iii) If $\nu \|_{*} \hat{\nu}$ and if $\hat{\nu}_{1} \geq_{*} \hat{\nu}$, then $\nu \|_{*} \hat{\nu}_{1}$.

Since we will be concerned with states $\nu$ of arbitrarily large length, the following notation will be useful:

Definition. Let $\nu$ and $\nu^{\prime}$ be states, and suppose $\nu=(e, \sigma, \tau)$ and $\nu^{\prime}=\left(e^{\prime}, \sigma^{\prime}, \tau^{\prime}\right)$. We put

(i) $\nu\lceil n=(n, \sigma \cap\{0,1, \ldots, n\}, \tau \cap\{0,1, \ldots, n\})$.

(ii) $\nu \preceq \nu^{\prime}$ if and only if $e \leq e^{\prime}$ and $\nu=\nu^{\prime} \uparrow e$.

Some more notions which we will use in the remainder of this paper: As usual, $\mathcal{L}$ is a list or sequence if $\mathcal{L}$ is a function on $\alpha$ where $\alpha \leq \omega$. By an occurrence of $\alpha$ in the sequence $\mathcal{L}$, we mean an integer $n$ such that $\mathcal{L}(n)=\alpha$. We say that $\mathcal{M}$ is a marked list if $\mathcal{M}$ is a pair $(\mathcal{L}, S)$ such that $\mathcal{L}$ is a list and $S \subseteq \operatorname{dom} \mathcal{L}$. We say that $n$ is marked on $\mathcal{M}$ if $n \in S$. If $\mathcal{M}=(\mathcal{L}, S)$ is a marked list, we put ${ }^{+} \mathcal{M}=\mathcal{L}$ and ${ }^{-} \mathcal{M}=S$. If $\mathcal{M}_{1}$ and $\mathcal{M}_{2}$ are marked lists, we put $\mathcal{M}_{1} \subseteq \mathcal{M}_{2}$ if ${ }^{+} \mathcal{M}_{1} \subseteq{ }^{+} \mathcal{M}_{2}$ and ${ }^{-} \mathcal{M}_{1} \subseteq-\mathcal{M}_{2}$. We say that $\mathcal{S}$ is a stream if $\mathcal{S}$ is a set of pairs $(\nu, x)$ such that $\nu$ is a state and $x \in \omega$.

Suppose $R \subseteq \omega$. We say that $R$ holds at stage $s$ if $R(s)$, that $R$ holds by stage $s$ if $R(t)$ for some $t \leq s$, and that $R$ holds infinitely often if $\{s \mid R(s)\}$ is infinite. Now suppose that $f$ is a function. Let $\alpha$ be arbitrary. We say that $f(\alpha)$ exists (written $f(\alpha) \downarrow$ ) if $\alpha \in \operatorname{dom} f$. If $f(\alpha)$ does not exist, we write $f(\alpha) \uparrow$. If $\operatorname{dom} f \subseteq \omega$ and $s \in \operatorname{dom} f$, we sometimes call $f(s)$ " $f$ at stage $s$ ".

Given any sets $X, Y$, and $y \in Y$, we put $X^{<\omega}=\bigcup_{n} X^{n}, F S(X)=\{D \mid$ $D$ is a finite subset of $X\}, F F(X, Y)=\{f \mid f: D \rightarrow Y$ for some finite $D \subseteq X\}$, and $F F_{y}(X, Y)=\{f \mid f: X \rightarrow Y$ and $f(x)=y$ for all but finitely many $x \in X\}$. ("FS" stands for "finite subset" and " $F F$ " stands for "finite function".) Let $J$ be the set of integers.

\section{THE ISOMORPHISM CONSTRUCTION}

In the next three sections we give our modification of Soare's automorphism construction, in order to show that $\left(\mathcal{E}^{A}\right)^{*} \cong_{1}\left(\mathcal{E}^{B}\right)^{*}$ (recall that $A$ and $B$ are sets such that $\left.A^{\prime} \equiv_{T} B^{\prime}\right)$.

For the remainder of this paper, fix a bijection (a "coding")

$$
\begin{aligned}
K: \omega \rightarrow & F F_{\emptyset}(\omega, F S(\omega))^{3} \times F S(\omega)^{9} \times F F_{\emptyset}(S, F S(\omega)) \times F S(S)^{2} \\
& \times\left(S^{<\omega} \times F S(\omega)\right) \times F F_{(\emptyset, \emptyset)}\left(S, S^{<\omega} \times F S(\omega)\right) \times F F\left(\omega^{2}, S\right) \\
& \times F F(S, \omega) \times F F(\omega, J) \times F S(S \times \omega)^{6} \times F F(S, \omega) \times \omega .
\end{aligned}
$$

(It is not hard to see that any two "natural" choices $K_{1}, K_{2}$ for $K$ are recursively equivalent in the sense that there is a recursive permutation $\pi$ of $\omega$ such that $K_{1}=K_{2} \circ \pi$, so the choice of $K$ is not particularly important. However, we will omit the definition of exactly what we mean by the term "natural" here.)

Fix a sequence ${ }^{0} \mathcal{R}$ of pairs $(\nu, i) \in S \times\{1,2\}$ such that (1) every pair $(\nu, i) \in$ $S \times\{1,2\}$ occurs infinitely often in ${ }^{0} \mathcal{R},(2){ }^{0} \mathcal{R}$ is recursive (i.e., recursive as a function from $\omega$ to $S \times \omega)$. Fix a recursive bijection $\langle-,-\rangle: \omega^{2} \rightarrow \omega$ and a $(1,1)$ ary recursive functional $g$ such that for all $Y \subseteq \omega, g^{Y}$ is one-one and has range exactly $\left\{\langle n, x\rangle \mid x \in W_{n}^{Y}\right\}$. (It is not hard to see that such a functional $g$ exists.) Let $W_{n, s}^{Y}=\left\{x \mid\langle n, x\rangle \in g^{Y}(t)\right.$ for some $\left.t<s\right\}$. We may assume that $W_{0}^{Y}=\omega$ and that $W_{n, s}^{Y} \subseteq W_{0, s}^{Y}$ for all $n$ and $s$. 
Fix $b \in \omega, v: \omega \rightarrow \omega, Y \subseteq \omega$. The intuition behind the parameters $b, v$ and $Y$ is as follows. We are defining one half of the construction, and this half is done relative to the "oracle" $Y$, either $A$ or $B$. Suppose for example that $Y=A$. Then $v$ is a recursive permutation $f$ (obtained using Proposition 1) such that for all $e$, $W_{e}^{B}$ is infinite if and only if $W_{f(e)}^{A}$ is infinite. $b$ is an index for the other half of the construction, in this case the half relative to the "oracle" $B$, and is obtained using Smullyan's Double Recursion Theorem. The values of $b, v$, and $Y$ will be defined precisely in Section 5.

The goal of this section is to define for each $s \in \omega$ the objects

$$
\left\{\begin{array}{l}
\left(U_{n, s}^{b, v}, Y\right)_{n \in \omega},\left(\hat{V}_{n, s}^{+b, v, Y}\right)_{n \in \omega}, H_{s}^{b, v, Y}, C_{s}^{b, v, Y}, C_{1, s}^{b, v, Y}, C_{2, s}^{b, v, Y}, D_{s}^{b, v, Y} \\
Q_{s}^{b, v, Y}, \mathcal{R}_{s}^{b, v, Y},\left(B_{\nu, s}^{b, v}\right)_{\nu \in S}, \mathcal{I}_{s}^{b, v, Y},\left(\mathcal{L}_{\nu, s}^{b, v}, Y\right)_{\nu \in S}, w_{s}^{b, v}, n_{s}^{b, v, Y} \\
\left(\hat{V}_{n, s}^{b, v}\right)_{n \in \omega}, P_{s}^{b, v, Y}, M_{s}^{b, v, Y}, \nu_{s}^{b, v, Y}, q_{s}^{b, v, Y}, \mathcal{S}_{s}^{b, v, Y}(C), \mathcal{S}_{s}^{b, v, Y}\left(C_{1}\right), \\
\mathcal{S}_{s}^{b, v, Y}\left(C_{2}\right), \mathcal{S}_{s}^{b, v, Y}(D), \mathcal{S}_{s}^{b, v, Y}(P), \mathcal{S}_{s}^{b, v, Y}(Q), \mathcal{M}_{s}^{b, v, Y}, \mathcal{P}_{s}^{b, v, Y}, d_{s}^{b, v, Y}
\end{array}\right.
$$

We define $\ddagger(s)$ to be the finite sequence of objects in (0). For each $s \in \omega$,

$$
\begin{gathered}
\lambda n U_{n, s}^{b, v, Y}, \lambda n \hat{V}_{n, s}^{+b, v, Y}, \lambda n \hat{V}_{n, s}^{b, v, Y} \in F F_{\emptyset}(\omega, F S(\omega)) ; \\
H_{s}^{b, v, Y}, C_{s}^{b, v, Y}, C_{1, s}^{b, v, Y}, C_{2, s}^{b, v, Y}, D_{s}^{b, v, Y}, P_{s}^{b, v, Y}, Q_{s}^{b, v, Y}, M_{s}^{b, v, Y},{ }^{1} \mathcal{R}_{s}^{b, v, Y} \in F S(\omega) ; \\
\lambda \nu B_{\nu, s}^{b, v, Y} \in F F_{\emptyset}(S, F S(\omega)) ; \quad \mathcal{M}_{s}^{b, v, Y}, \mathcal{P}_{s}^{b, v, Y} \in F S(S) ; \\
\mathcal{I}_{s}^{b, v, Y} \in S^{<\omega} \times F S(\omega) ; \quad \lambda \nu \mathcal{L}_{\nu, s}^{b, v, Y} \in F F_{(\emptyset, \emptyset)}\left(S, S^{<\omega} \times F S(\omega)\right) ; \\
\nu_{s}^{b, v, Y} \in F F\left(\omega^{2}, S\right) ; \quad q_{s}^{b, v, Y} \in F F(S, \omega) ; \quad d_{s}^{b, v, Y} \in F F(\omega, J) ; \\
\mathcal{S}_{s}^{b, v, Y}(C), \mathcal{S}_{s}^{b, v, Y}\left(C_{1}\right), \mathcal{S}_{s}^{b, v, Y}\left(C_{2}\right), \mathcal{S}_{s}^{b, v, Y}(D), \mathcal{S}_{s}^{b, v, Y}(P), \mathcal{S}_{s}^{b, v, Y}(Q) \in F S(S \times \omega) ; \\
w_{s}^{b, v, Y} \in F F(S, \omega) ; \quad n_{s}^{b, v, Y} \in \omega .
\end{gathered}
$$

In Section 4.2, we will define the function $\lambda s \ddagger(s)$ by course of values induction.

We drop the superscripts $b, v, Y$ for the remainder of this section.

Remark. The reader may find it helpful to think of $M_{s}$ as the set of balls in a "pinball machine" at "stage" $s$, where a stage is just an element of $\omega$. Then $H$ is a "hole" of the pinball machine, $C, C_{1}, C_{2}$, and $D$ are "tracks", $P$ and $Q$ are "pockets", and each $B_{\nu}(\nu \in S)$ is a "box". The reader may find Figure 1 (from Diagram 6.1 of Soare [9], p. 361) helpful in visualizing this "pinball machine". Part of the work in Section 4.2 can then be thought of as moving a ball from one hole, track, box, or pocket to another.

4.1. Preliminaries. We now make some definitions which we will need in Section 4.2 for the definition of the function $\lambda s \ddagger(s)$.

We say that $e$ is verified at stage $s$ if $W_{v(e), s}^{Y}-W_{v(e), s-1}^{Y} \neq \emptyset$ (where we put $\left.W_{v(e),-1}^{Y}=\emptyset\right)$. We say that $e$ has been verified $u$ times by stage $s$ if $\left|W_{v(e), s}^{Y}\right| \geq u$.

Recall the coding $K$ chosen at the beginning of the section. If $Z \subseteq \omega, s \in \omega$, and $\{b\}^{Z}(s) \downarrow, K\left(\{b\}^{Z}(s)\right)$ is a 28-tuple. We define $\left(V_{n, s}^{Z}\right)_{n \in \omega},\left(\hat{U}_{n, s}^{+Z}\right)_{n \in \omega},\left(\hat{U}_{n, s}^{Z}\right)_{n \in \omega}$, $\hat{H}_{s}^{Z}, \hat{C}_{s}^{Z}, \hat{C}_{1, s}^{Z}, \hat{C}_{2, s}^{Z}, \hat{D}_{s}^{Z}, \hat{Q}_{s}^{Z}, \hat{P}_{s}^{Z}, \hat{M}_{s}^{Z},{ }^{1} \hat{\mathcal{R}}_{s}^{Z},\left(\hat{B}_{\nu, s}^{Z}\right)_{\nu \in S}, \hat{\mathcal{M}}_{s}^{Z}, \hat{\mathcal{P}}_{s}^{Z}, \hat{\mathcal{I}}_{s}^{Z},\left(\hat{\mathcal{L}}_{\nu, s}^{Z}\right)_{\nu \in S}$, $\hat{\nu}_{s}^{Z}, \hat{q}_{s}^{Z}, \hat{d}_{s}^{Z}, \mathcal{S}_{s}\left(\hat{C}^{Z}\right), \mathcal{S}_{s}\left(\hat{C}_{1}^{Z}\right), \mathcal{S}_{s}\left(\hat{C}_{2}^{Z}\right), \mathcal{S}_{s}\left(\hat{D}^{Z}\right), \mathcal{S}_{s}\left(\hat{P}^{Z}\right), \mathcal{S}_{s}\left(\hat{Q}^{Z}\right), \hat{w}_{s}^{Z}$, and $\hat{n}_{s}^{Z}$ to be the 1st, 2nd, .., 28th terms of the sequence $K\left(\{b\}^{Z}(s)\right)$. 
FiguRE 1. Machine $M$ [From R. I. Soare, Recursively enumerable sets and degrees: a study of computable functions and computably generated sets, Springer-Verlag, Berlin, Heidelberg, New York, London, Paris, Tokyo, 1987, p. 361. Used with permission.]

We use the symbol $X$ to range over the symbols $C, C_{1}, C_{2}, D, P, Q$ in the following sense: $(\exists X)[\cdots X \cdots]$ is an abbreviation for $(\cdots C \cdots) \vee\left(\cdots C_{1} \cdots\right) \vee\left(\cdots C_{2} \cdots\right) \vee$ $(\cdots D \cdots) \vee(\cdots P \cdots) \vee(\cdots Q \cdots)$. 
We also need the following easy proposition, whose proof we omit:

Proposition 1. There are recursive functions $\alpha, \beta, \gamma, \delta, \epsilon, \zeta: S \rightarrow \omega$ such that for all states $\hat{\mu}$ and all $Z \subseteq \omega$,

$$
\begin{gathered}
W_{\alpha(\hat{\mu})}^{Z}=\left\{\hat{y} \mid(\exists s)\left[\{b\}^{Z}(s) \downarrow \wedge(\hat{\mu}, \hat{y}) \in \mathcal{S}_{s}\left(\hat{D}^{Z}\right)\right]\right\}, \\
W_{\beta(\hat{\mu})}^{Z}=\left\{s \mid\{b\}^{Z}(s) \downarrow \wedge \hat{B}_{\hat{\mu}, s}^{Z}=\emptyset\right\}, \\
W_{\gamma(\hat{\mu})}^{Z}=\left\{s \mid\{b\}^{Z}(s) \downarrow \wedge\left[\hat{q}_{s}^{Z}(\hat{\mu}) \uparrow \vee\right.\right. \\
\left.\left.(\exists t>s)\left[\{b\}^{Z}(t) \downarrow \wedge\left(\hat{q}_{t}^{Z}(\hat{\mu}) \uparrow \vee \hat{q}_{t}^{Z}(\hat{\mu}) \neq \hat{q}_{s}^{Z}(\hat{\mu})\right)\right]\right]\right\}, \\
W_{\delta(\hat{\mu})}^{Z}=\left\{s \mid(\forall t \leq s)\left[\{b\}^{Z}(t) \downarrow\right] \wedge(\exists \hat{y})(\exists X)\left[\hat{\nu}_{s}^{Z}(|\hat{\mu}|, \hat{y}) \downarrow\right.\right. \\
\wedge\left(\hat{\nu}_{s}^{Z}(|\hat{\mu}|, \hat{y}), \hat{y}\right) \in \mathcal{S}_{s}\left(\hat{X}^{Z}\right) \wedge \hat{\nu}_{s}^{Z}(|\hat{\mu}|, \hat{y}) \notin \hat{\mathcal{P}}_{s} \\
\left.\left.\wedge(\exists t \leq s)\left[\hat{\nu}_{t}^{Z}(|\hat{\mu}|, \hat{y}) \downarrow \wedge \hat{\mu}=\hat{\nu}_{t}^{Z}(|\hat{\mu}|, \hat{y})\right]\right]\right\}, \\
W_{\epsilon(\hat{\mu})}^{Z}=\left\{s \mid\{b\}^{Z}(s) \downarrow \wedge(\exists \hat{y})(\exists X)\left[(\hat{\mu}, \hat{y}) \in \mathcal{S}_{s}\left(\hat{X}^{Z}\right)\right]\right\}, \\
W_{\zeta(\hat{\mu})}^{Z}=\left\{s \mid\{b\}^{Z}(s) \downarrow \wedge(\exists \hat{y})\left[(\hat{\mu}, \hat{y}) \in \mathcal{S}_{s}\left(\hat{D}^{Z}\right)\right]\right\} .
\end{gathered}
$$

Now fix recursive functions $\alpha, \beta, \gamma, \delta, \epsilon, \zeta$ as in the statement of the proposition.

4.2. Definition of $\lambda s \ddagger(s)$. We now define the function $\lambda s \ddagger(s)$ by course of values induction. At "stage $s$ " we will define $\ddagger(s)$ from $(\ddagger(t))_{t<s}$, in such a way that $(1)_{s}$ if $s>0$, then for all $n \in \omega$ and $\nu \in S, U_{n, s} \supseteq U_{n, s-1}, \hat{V}_{n, s} \supseteq \hat{V}_{n, s-1},{ }^{1} \mathcal{R}_{s} \supseteq{ }^{1} \mathcal{R}_{s-1}$, $\mathcal{I}_{s} \supseteq \mathcal{I}_{s-1}$, and $\mathcal{L}_{\nu, s} \supseteq \mathcal{L}_{\nu, s-1} ;(2)_{s} C_{s} \cup C_{1, s} \cup C_{2, s} \cup D_{s}$ has at most one element; $(3)_{s}$ the sets $H_{s}, C_{s}, C_{1, s}, C_{2, s}, D_{s}, Q_{s}$ and $B_{\nu, s}(\nu \in S)$ are mutually disjoint; (4) ${ }_{s} P_{s}=\bigcup_{\nu} B_{\nu, s} ;(5)_{s} M_{s}=H_{s} \cup C_{s} \cup C_{1, s} \cup C_{2, s} \cup D_{s} \cup P_{s} \cup Q_{s}$; (6) $s$ (a) for all $e, x \in \omega,(e, x) \in \operatorname{dom} \nu_{s}$ if and only if $e \leq x$ and $x \in M_{s}$, and (b) for all $(e, x) \in \operatorname{dom} \nu_{s}, \nu_{s}(e, x)=\left(e,\left\{i \leq e \mid x \in U_{i, s}\right\},\left\{i \leq e \mid x \in \hat{V}_{i, s}\right\}\right) ;(7)_{s}$ $\mathcal{P}_{s}=\left\{\nu \mid\left(\exists \hat{\nu} \in \mathcal{M}_{s}\right)\left[\hat{\nu}_{*} \| \nu\right]\right\} ;(8)_{s}$ (a) for all $y \in \omega, y \in \operatorname{dom} d_{s}$ if and only if $y \in M_{s}$, and (b) for all $y \in \operatorname{dom} d_{s}$, either $d_{s}(y)<0$ or $\nu_{s}\left(d_{s}(y), y\right) \in \mathcal{P}_{s}$.

So assume that we are given $(\ddagger(t))_{t<s}$ and that for all $t<s,(1)_{t}-(8)_{t}$ hold. In the remainder of this subsection, we define $\ddagger(s)$ and show that $(1)_{s}-(8)_{s}$ hold.

4.2.1. Definition of $\left(U_{n, s}\right)_{n \in \omega},\left(\hat{V}_{n, s}^{+}\right)_{n \in \omega}, H_{s}, C_{s}, C_{1, s}, C_{2, s}, D_{s},\left(B_{\nu, s}\right)_{\nu \in S}, Q_{s}$, ${ }^{1} \mathcal{R}_{s}, \mathcal{I}_{s},\left(\mathcal{L}_{\nu, s}\right)_{\nu \in S}, w_{s}, n_{s}$. We define these elements by cases:

Case 0: $s=0$. For all $\nu \in S$ and $n \in \omega$, put $U_{n, s}=\hat{V}_{n, s}^{+}=H_{s}=C_{s}=C_{1, s}=$ $C_{2, s}=D_{s}=B_{\nu, s}=Q_{s}={ }^{1} \mathcal{R}_{s}=w_{s}=\emptyset$, put $\mathcal{I}_{s}=\mathcal{L}_{\nu, s}=(\emptyset, \emptyset)$, and put $n_{s}=0$.

Case 1: $s>0$ and some element $x$ is in $C_{s-1} \cup C_{1, s-1} \cup C_{2, s-1} \cup D_{s-1}$. By $(2)_{s-1}$, the element $x$ is unique. Put $U_{n, s}=U_{n, s-1}$ and $n_{s}=n_{s-1}$. If $x \in C_{s-1}$, then

"Rule $R_{1}$ ". Let $n \in \omega$ be the least $n$ such that $n \notin{ }^{1} \mathcal{R}_{s-1}$ and such that, letting $(\mu, i)={ }^{0} \mathcal{R}(n), \mu \preceq \nu_{s-1}(x, x)$. If $i=1$, put $C_{1, s}=\{x\}$ and put $C_{2, s}=\emptyset$, and if $i=2$, put $C_{2, s}=\{x\}$ and put $C_{1, s}=\emptyset$. Put ${ }^{1} \mathcal{R}_{s}={ }^{1} \mathcal{R}_{s-1} \cup\{n\}$, $C_{s}=D_{s}=w_{s}=\emptyset, H_{s}=H_{s-1}, Q_{s}=Q_{s-1}, B_{\nu, s}=B_{\nu, s-1}$ for all $\nu, \mathcal{I}_{s}=\mathcal{I}_{s-1}$, $\mathcal{L}_{\nu, s}=\mathcal{L}_{\nu, s-1}$ for all $\nu$, and $\hat{V}_{n, s}^{+}=\hat{V}_{n, s-1}$ for all $n$.

If $x \in D_{s-1}$, then

"Rule $R_{2}$ ". Let $u$ be the largest $u<s$ such that $D_{u} \neq \emptyset$, if there is such a $u$, and 0 otherwise.

Step 1. For each state $\nu$ define a set $B_{\nu}^{+}$as follows: If $\gamma\left(\nu^{*}\right)$ is verified at some stage $t$ with $u<t<s$, then put $B_{\nu}^{+}=\emptyset$. Otherwise, put $B_{\nu}^{+}=B_{\nu, s-1}$.

Step 2. Define $\Delta=\bigcup_{\nu}\left(B_{\nu, s-1}-B_{\nu}^{+}\right)$. 
Step 3. For each $y \in \Delta$, let $t_{y}$ be the largest $t<s$ such that $y \notin P_{t}$. Put $H_{s}=H_{s-1} \cup\left\{y \in \Delta \mid \nu_{t_{y}}(y, y) \neq \nu_{s-1}(y, y)\right\}$ and define $Q^{+}=Q_{s-1} \cup\{y \in \Delta \mid$ $\left.\nu_{t_{y}}(y, y)=\nu_{s-1}(y, y)\right\}$.

Step 4. For each $\nu$ such that $B_{\nu, s-1}=\emptyset$, let ${ }^{+} \mathcal{L}_{\nu, s}$ be ${ }^{+} \mathcal{L}_{\nu, s-1}$ concatenated with the sequence of states $\mu$ (listed, say, in the order of their codes) such that $\nu \preceq \mu$ and $|\mu|<s$ and such that there is no unmarked occurrence of $\mu$ on $\mathcal{L}_{\nu, s-1}$. For each $\nu$ such that $B_{\nu, s-1} \neq \emptyset$, let ${ }^{+} \mathcal{L}_{\nu, s}={ }^{+} \mathcal{L}_{\nu, s-1}$.

Step 5. If there are states $\pi$ and $\mu$ such that $\pi \preceq \mu \preceq \nu_{s-1}(x, x)$ and $\mu$ occurs unmarked on $\mathcal{L}_{\pi, s}$, then choose the $\pi$ of least length and then the $\mu$ as above which first occurs unmarked on $\mathcal{L}_{\pi, s}$, and put $B_{\pi, s}=B_{\pi}^{+} \cup\{x\}, B_{\nu, s}=B_{\nu}^{+}$for all $\nu \neq \pi, Q_{s}=Q^{+},{ }^{-} \mathcal{L}_{\pi, s}={ }^{-} \mathcal{L}_{\pi, s-1} \cup\left({ }^{+} \mathcal{L}_{\pi, s}\right)^{-1}[\mu]$, and ${ }^{-} \mathcal{L}_{\nu, s}={ }^{-} \mathcal{L}_{\nu, s-1}$ for all $\nu \neq \pi$. If no such $\pi$ and $\mu$ exist, put $B_{\nu, s}=B_{\nu}^{+}$for all $\nu$, put $Q_{s}=Q^{+} \cup\{x\}$, and let $-\mathcal{L}_{\nu, s}={ }^{-} \mathcal{L}_{\nu, s-1}$ for all $\nu$. In either case, put $C_{s}=C_{1, s}=C_{2, s}=D_{s}=$ $w_{s}=\emptyset, H_{s}=H_{s-1},{ }^{1} \mathcal{R}_{s}={ }^{1} \mathcal{R}_{s-1}$, and $\mathcal{I}_{s}=\mathcal{I}_{s-1}$, and $\hat{V}_{n, s}^{+}=\hat{V}_{n, s-1}$ for all $n$. If $x \in C_{2, s-1}$, then

"Rule $R_{3}$ ". Step 1. Define $w_{s}$ by putting $\hat{\nu} \in \operatorname{dom} w_{s}$ if and only if $\hat{\nu}$ is of length $\leq s$ and $\hat{\nu}$ does not occur unmarked on $\mathcal{I}_{s-1}$, and by for each such $\hat{\nu}$ letting $w_{s}(\hat{\nu})$ be the largest $t<s-1$ such that $\hat{\nu}$ occurs unmarked in $\mathcal{I}_{t}$, if such a $t$ exists, and $|\hat{\nu}|$ otherwise.

Step 2. Let ${ }^{+} \mathcal{I}_{s}$ be ${ }^{+} \mathcal{I}_{s-1}$ concatenated with the sequence of states $\hat{\nu}$ (listed, say, in the order of their codes) such that $\hat{\nu} \in \operatorname{dom} w_{s}$ and such that $\delta(\hat{\nu})$ and $\epsilon(\hat{\mu})$ for all $\hat{\mu} \preceq \hat{\nu}$ have been verified $w_{s}(\hat{\nu})$ times by stage $s-1$.

Step 3. If there is a $\hat{\pi}$ such that $\hat{\pi} \| \nu_{s-1}(|\hat{\pi}|, x)$ and $\hat{\pi}$ occurs unmarked on $\left({ }^{+} \mathcal{I}_{s}, \mathcal{I}_{s-1}\right)$, then choose the $\hat{\pi} \| \nu_{s-1}(|\hat{\pi}|, x)$ which first occurs unmarked on $\left({ }^{+} \mathcal{I}_{s},{ }^{-} \mathcal{I}_{s-1}\right)$. Suppose that $\hat{\pi}=(e, \tau, \hat{\sigma})$ and $\nu_{s-1}(e, x)=(e, \sigma, \hat{\tau})$. Put $\hat{V}_{i, s}^{+}=\hat{V}_{i, s-1} \cup\{x\}$ for all $i \in \tau-\hat{\tau}$, put $\hat{V}_{i, s}^{+}=\hat{V}_{i, s-1}$ for all $i \notin \tau-\hat{\tau}$, and put ${ }^{-} \mathcal{I}_{s}={ }^{-} \mathcal{I}_{s-1} \cup\left({ }^{+} \mathcal{I}_{s}\right)^{-1}[\hat{\pi}]$. If there is no such $\hat{\pi}$, put $\hat{V}_{i, s}^{+}=\hat{V}_{i, s-1}$ for all $i$, and put $\mathcal{I}_{s}=\mathcal{I}_{s-1}$. Put $C_{s}=C_{1, s}=C_{2, s}=\emptyset, D_{s}=\{x\}, H_{s}=H_{s-1}, Q_{s}=Q_{s-1}$, $B_{\nu, s}=B_{\nu, s-1}$ for all $\nu,{ }^{1} \mathcal{R}_{s}={ }^{1} \mathcal{R}_{s-1}$, and $\mathcal{L}_{\nu, s}=\mathcal{L}_{\nu, s-1}$ for all $\nu$.

Otherwise $x \in C_{1, s-1}$ : then put $C_{s}=C_{1, s}=C_{2, s}=w_{s}=\emptyset, D_{s}=\{x\}, H_{s}=$ $H_{s-1}, Q_{s}=Q_{s-1}, B_{\nu, s}=B_{\nu, s-1}$ for all $\nu,{ }^{1} \mathcal{R}_{s}={ }^{1} \mathcal{R}_{s-1}, \mathcal{I}_{s}=\mathcal{I}_{s-1}, n_{s}=n_{s-1}$, $\mathcal{L}_{\nu, s}=\mathcal{L}_{\nu, s-1}$ for all $\nu$, and $\hat{V}_{n, s}^{+}=\hat{V}_{n, s-1}$ for all $n$.

Case 2: $s>0, C_{s-1} \cup C_{1, s-1} \cup C_{2, s-1} \cup D_{s-1}=\emptyset$, and $H_{s-1} \neq \emptyset$. Let $x$ be the least element in $H_{s-1}$. Put $H_{s}=H_{s-1}-\{x\}$ and $C_{s}=\{x\}$. Put $C_{1, s}=C_{2, s}=$ $D_{s}=w_{s}=\emptyset$, put $Q_{s}=Q_{s-1}, B_{\nu, s}=B_{\nu, s-1}$ for all $\nu,{ }^{1} \mathcal{R}_{s}={ }^{1} \mathcal{R}_{s-1}, \mathcal{I}_{s}=\mathcal{I}_{s-1}$, and $\mathcal{L}_{\nu, s}=\mathcal{L}_{\nu, s-1}$ for all $\nu$. Put $U_{n, s}=U_{n, s-1}$ and $\hat{V}_{n, s}^{+}=\hat{V}_{n, s-1}$ for all $n$, and put $n_{s}=n_{s-1}$.

Case 3: $s>0, C_{s-1} \cup C_{1, s-1} \cup C_{2, s-1} \cup D_{s-1}=\emptyset$, and $H_{s-1}=\emptyset$. Let $(e, x)=$ $g^{Y}\left(n_{s-1}\right)$. Put $n_{s}=n_{s-1}+1, C_{s}=C_{1, s}=C_{2, s}=D_{s}=\emptyset,{ }^{1} \mathcal{R}_{s}={ }^{1} \mathcal{R}_{s-1}$, $\mathcal{I}_{s}=\mathcal{I}_{s-1}$, and $\mathcal{L}_{\nu, s}=\mathcal{L}_{\nu, s-1}$ for all $\nu$. Put $\hat{V}_{n, s}^{+}=\hat{V}_{n, s-1}$ for all $n$. Then:

Case 3A: $x<e$. Put $U_{n, s}=U_{n, s-1}$ for all $n, H_{s}=\emptyset, Q_{s}=Q_{s-1}$, and $B_{\nu, s}=B_{\nu, s-1}$ for all states $\nu$.

Case 3B: $x \geq e$. Put $U_{e, s}=U_{e, s-1} \cup\{x\}$ and $U_{n, s}=U_{n, s-1}$ for all $n \neq e$. Then Case 3B1: $e=0$. Put $H_{s}=\{x\}$ and $Q_{s}=Q_{s-1}$, and put $B_{\nu, s}=B_{\nu, s-1}$ for all states $\nu$.

Case 3B2: $e>0$ and $x \in P_{s-1}$. Let $\mu$ denote the state such that $x \in B_{\mu, s-1}$; from $(3)_{s-1}$ and $(4)_{s-1}$ there is exactly one such state. If $e \leq|\mu|$, put $B_{\mu, s}=$ 
$B_{\mu, s-1}-\{x\}$ and put $H_{s}=\{x\}$. Otherwise, put $B_{\mu, s}=B_{\mu, s-1}$ and $H_{s}=\emptyset$. Put $B_{\nu, s}=B_{\nu, s-1}$ for all states $\nu \neq \mu$ and put $Q_{s}=Q_{s-1}$.

Case 3B3: $e>0$ and $x \notin P_{s-1}$. Put $Q_{s}=Q_{s-1}-\{x\}$ and $H_{s}=\{x\}$. Put $B_{\nu, s}=B_{\nu, s-1}$ for all states $\nu$.

The reader can easily verify that in all cases, $(1)_{s}-(3)_{s}$ hold.

4.2.2. Definition of $\left(\hat{V}_{n, s}\right)_{n \in \omega}$. If $s=0$, let $\hat{V}_{n, s}=\emptyset$ for all $n \in \omega$. If $s>0$, define the $\hat{V}_{n, s}(n \in \omega)$ by Rule $R_{4}$ :

"Rule $R_{4}$ ". Step 1. For each $y$ which is in $Q_{s} \cap Q_{s-1}$, define a set $K_{y}$ as follows: Let $e=d_{s-1}(y)$. If $e<0$ or if $\nu_{s-1}(e, y)^{*} \in \mathcal{M}_{s-1}$ then let $K_{y}=\emptyset$. Otherwise (using $(7)_{s-1}$ and $\left.(8)_{s-1}\right)$ there is some $\hat{\nu}_{y} \in \mathcal{M}_{s-1}$ of length $e$ such that $\hat{\nu}_{y *} \| \nu_{s-1}(e, y)$. Choose from among these $\hat{\nu}_{y}$ a $\hat{\nu}_{y}$ such that $\left\{t<s \mid \zeta\left(\hat{\nu}_{y}\right)\right.$ is verified at stage $\left.t\right\}$ has maximal cardinality. Suppose $\hat{\nu}_{y}=(e, \tau, \hat{\sigma})$ and $\nu_{s-1}(e, y)=(e, \sigma, \hat{\tau})$. Put $K_{y}=\tau-\hat{\tau}$.

Step 2. For each $i$, put $\hat{V}_{i, s}=\hat{V}_{i, s}^{+} \cup\left\{y \in Q_{s} \cap Q_{s-1} \mid i \in K_{y}\right\}$.

4.2.3. Definition of $P_{s}, M_{s}, \nu_{s}$. Put $P_{s}=\bigcup_{\nu} B_{\nu, s}$ and $M_{s}=H_{s} \cup C_{s} \cup C_{1, s} \cup C_{2, s} \cup$ $D_{s} \cup P_{s} \cup Q_{s}$. For all $e$ and $x \in \omega$, put $(e, x) \in \operatorname{dom} \nu_{s}$ if and only if $e \leq x$ and $x \in M_{s}$, in which case put $\nu_{s}(e, x)=\left(e,\left\{i \leq e \mid x \in U_{i, s}\right\},\left\{i \leq e \mid x \in \hat{V}_{i, s}\right\}\right)$. We say that $x$ has state $\mu$ at stage $s$ if $\nu_{s}(|\mu|, x) \downarrow$ and $\mu=\nu_{s}(|\mu|, x)$. (Clearly $(4)_{s}-(6)_{s}$ hold.)

4.2.4. Definition of $q_{s}$. To define $q_{s}$ we first define a function $g: S \rightarrow J$ by $\prec-$ induction (notice that the relation $\prec$ is a well-founded relation on states). Given $g\left(\nu^{\prime}\right)$ for all $\nu^{\prime} \prec \nu$, define $g(\nu)$ : if there is a $y \in Q_{s}$ such that $y$ has state $\nu$ at stage $s$, but such that for all $\nu^{\prime} \prec \nu, g\left(\nu^{\prime}\right) \neq y$, then let $g(\nu)$ be the least such $y$; otherwise, let $g(\nu)=-1$. Now define $q_{s}$ by: $\nu \in \operatorname{dom} q_{s}$ if and only if $g(\nu) \geq 0$, in which case $q_{s}(\nu)=g(\nu)$.

4.2.5. Definition of $\mathcal{S}_{s}(C), \mathcal{S}_{s}\left(C_{1}\right), \mathcal{S}_{s}\left(C_{2}\right), \mathcal{S}_{s}(D), \mathcal{S}_{s}(P), \mathcal{S}_{s}(Q)$. If $X$ is one of the symbols $C, C_{1}, C_{2}$, or $D$, then we put $\mathcal{S}_{s}(X)=\left\{(\nu, x) \in S \times \omega \mid x \in X_{s} \wedge \nu \preceq\right.$ $\left.\nu_{s}(x, x)\right\}$, and if $X$ is one of the symbols $P$ or $Q$, then we put $\mathcal{S}_{s}(X)=\{(\nu, x) \in$ $\left.S \times \omega \mid x \in X_{s} \wedge \nu \preceq \nu_{s}(x, x) \wedge\left(s=0 \vee x \notin X_{s-1} \vee \nu_{s}(x, x) \neq \nu_{s-1}(x, x)\right)\right\}$.

4.2.6. Definition of $\mathcal{M}_{s}, \mathcal{P}_{s}$. We will first define two special properties, called Condition (a) and Condition (b). If $\hat{\nu}$ is a state and $r<s$, then we say that Condition (a) holds of $\hat{\nu}$ at stage $r$ if $(\exists \nu)(\exists x)(\exists X)\left[|\nu|<|\hat{\nu}| \wedge(\nu, x) \in \mathcal{S}_{r}(X) \wedge \nu \notin \mathcal{P}_{r}\right]$. To define Condition (b), first define for each state $\hat{\nu}$ and each $r<s$ an element $u_{\hat{\nu}, r}$ of $\omega$ as follows: if there is a $u<r$ such that $\hat{\nu} \in \mathcal{M}_{u+1}-\mathcal{M}_{u}$, then let $u_{\hat{\nu}, r}$ be the largest such $u$, and put $u_{\hat{\nu}, r}=0$ otherwise. If $\hat{\nu}$ is a state and $r<s$, then we say that Condition (b) holds of $\hat{\nu}$ at stage $r$ if for some $\hat{\mu} \preceq \hat{\nu}, \beta(\hat{\mu})$ has been verified $u_{\hat{\nu}, r}$ times by stage $r$ and yet $q_{|\hat{\nu}|}\left(\hat{\mu}^{*}\right)=q_{t}\left(\hat{\mu}^{*}\right)$ for all $t$ with $|\hat{\nu}| \leq t \leq r$.

If $s=0$, we put $\mathcal{M}_{s}=\emptyset$. Otherwise, we define for each state $\hat{\nu}$ an element $t_{\hat{\nu}}$ of $\omega$ : if there is a $t<s$ such that $\hat{\nu} \in \mathcal{M}_{t}$, then we let $t_{\hat{\nu}}$ be the largest such $t$, and we let $t_{\hat{\nu}}=|\hat{\nu}|$ otherwise. Define $\mathcal{M}_{s}=\left\{\hat{\nu} \in S \mid\left(\hat{\nu} \in \mathcal{M}_{s-1}\right.\right.$ and neither Condition (a) nor Condition (b) holds of $\hat{\nu}$ at stage $s-1) \vee\left(\hat{\nu} \notin \mathcal{M}_{s-1}\right.$ and for all $\hat{\mu} \preceq \hat{\nu}, \alpha(\hat{\mu})$ has been verified $t_{\hat{\nu}}$ times by stage $\left.\left.s-1\right)\right\}$. Put $\mathcal{P}_{s}=\left\{\nu \mid\left(\exists \hat{\nu} \in \mathcal{M}_{s}\right)[\hat{\nu} * \| \nu]\right\}$. (Thus $(7)_{s}$ holds.) 
4.2.7. Definition of $d_{s}$. We define $d_{s}$ by putting $y \in \operatorname{dom} d_{s}$ if and only if $y \in M_{s}$, in which case we let $d_{s}(y)$ be the largest $e \in \omega \cup\{-1\}$ such that both (1) either (a) $e=-1$ or (b) $e \leq y$ and $\nu_{s}(e, y) \in \mathcal{P}_{s}$, and (2) if $s>0$ and $y \in M_{s-1}$, then $d_{s-1}(y) \geq e$. (It is easy to see by the definition of $d_{s}$ that $(8)_{s}$ holds.)

This completes the definition of the function $\lambda s \ddagger(s)$.

\section{Definition of the isomorphism}

Given $n, k \in \omega$ and a $(1, n+1)$-ary partial functional $H$, let $H_{k}$ be as defined in Section 1. We use Smullyan's Double Recursion Theorem $[6]$ in the following form:

Theorem. Fix $n \in \omega$, let $F, G$ be $(1, n+1)$-ary partial recursive functionals, and let $\{e\}$ be the e-th $(1, n)$-ary partial recursive functional. Then there are $a, b \in \omega$ such that $F_{b}=\{a\}$ and $G_{a}=\{b\}$.

This differs from the usual form of Smullyan's Double Recursion Theorem only in that set variables are allowed. Otherwise, the proof is as usual (cf. Soare [9], pp. $39-40)$.

Recall that $A, B$ are sets such that $A^{\prime} \equiv_{T} B^{\prime}$. Apply Proposition 2.1 to get a recursive permutation $f$ of $\omega$ such that for all $e \in \omega, W_{e}^{B}$ is infinite if and only if $W_{f(e)}^{A}$ is infinite. For convenience, put $g=f^{-1}$.

For each $b \in \omega, v: \omega \rightarrow \omega, Y \subseteq \omega$, define $N^{b, v, Y}: \omega \rightarrow \omega$ by putting for all $s \in \omega$

$$
\begin{aligned}
N^{b, v, Y}(s)=K^{-1}( & \left(U_{n, s}^{b, v, Y}\right)_{n \in \omega},\left(\hat{V}_{n, s}^{+b, v, Y}\right)_{n \in \omega},\left(\hat{V}_{n, s}^{b, v, Y}\right)_{n \in \omega}, H_{s}^{b, v, Y}, C_{s}^{b, v, Y}, \\
& C_{1, s}^{b, v, Y}, C_{2, s}^{b, v, Y}, D_{s}^{b, v, Y}, Q_{s}^{b, v, Y}, P_{s}^{b, v, Y}, M_{s}^{b, v, Y},{ }^{1} \mathcal{R}_{s}^{b, v, Y}, \\
& \left(B_{\nu, s}^{b, v, Y}\right)_{\nu \in S}, \mathcal{M}_{s}^{b, v, Y}, \mathcal{P}_{s}^{b, v, Y}, \mathcal{I}_{s}^{b, v, Y},\left(\mathcal{L}_{\nu, s}^{b, v, Y}\right)_{\nu \in S}, \nu_{s}^{b, v, Y}, \\
& q_{s}^{b, v, Y}, d_{s}^{b, v, Y}, \mathcal{S}_{s}^{b, v, Y}(C), \mathcal{S}_{s}^{b, v, Y}\left(C_{1}\right), \mathcal{S}_{s}^{b, v, Y}\left(C_{2}\right), \\
& \left.\mathcal{S}_{s}^{b, v, Y}(D), \mathcal{S}_{s}^{b, v, Y}(P), \mathcal{S}_{s}^{b, v, Y}(Q), w_{s}^{b, v, Y}, n_{s}^{b, v, Y}\right) .
\end{aligned}
$$

The reader can easily verify that there exist $(1,2)$-ary recursive functionals $F$ and $G$ such that for all $a, b, s \in \omega$ and all $Y \subseteq \omega, F(Y, b, s)=N^{b, f, Y}(s)$ and $G(Y, a, s)=$ $N^{a, g, Y}(s)$. Fix such functionals $F$ and $G$. Apply the Double Recursion Theorem to find elements $a, b \in \omega$ such that $F_{b}=\{a\}$ and $G_{a}=\{b\}$. So in particular, $N^{b, f, A}=\{a\}^{A}$ and $N^{a, g, B}=\{b\}^{B}$.

For all $n, s \in \omega$ and all states $\nu$, define $U_{n, s}=U_{n, s}^{b, f, A}, \hat{V}_{n, s}^{+}=\hat{V}_{n, s}^{+b, f, A}, \hat{V}_{n, s}=$ $\hat{V}_{n, s}^{b, f, A}, H_{s}=H_{s}^{b, f, A}, C_{s}=C_{s}^{b, f, A}, C_{1, s}=C_{1, s}^{b, f, A}, C_{2, s}=C_{2, s}^{b, f, A}, D_{s}=D_{s}^{b, f, A}$, $B_{\nu, s}=B_{\nu, s}^{b, f, A}, P_{s}=P_{s}^{b, f, A}, Q_{s}=Q_{s}^{b, f, A}, M_{s}=M_{s}^{b, f, A}, \mathcal{M}_{s}=\mathcal{M}_{s}^{b, f, A}, \mathcal{P}_{s}=$ $\mathcal{P}_{s}^{b, f, A},{ }^{1} \mathcal{R}_{s}={ }^{1} \mathcal{R}_{s}^{b, f, A}, \mathcal{I}_{s}=\mathcal{I}_{s}^{b, f, A}, \mathcal{L}_{\nu, s}=\mathcal{L}_{\nu, s}^{b, f, A}, \nu_{s}=\nu_{s}^{b, f, A}, q_{s}=q_{s}^{b, f, A}, d_{s}=$ $d_{s}^{b, f, A}, \mathcal{S}_{s}(C)=\mathcal{S}_{s}^{b, f, A}(C), \mathcal{S}_{s}\left(C_{1}\right)=\mathcal{S}_{s}^{b, f, A}\left(C_{1}\right), \mathcal{S}_{s}\left(C_{2}\right)=\mathcal{S}_{s}^{b, f, A}\left(C_{2}\right), \mathcal{S}_{s}(D)=$ $\mathcal{S}_{s}^{b, f, A}(D), \mathcal{S}_{s}(P)=\mathcal{S}_{s}^{b, f, A}(P), \mathcal{S}_{s}(Q)=\mathcal{S}_{s}^{b, f, A}(Q), w_{s}=w_{s}^{b, f, A}$, and $n_{s}=n_{s}^{b, f, A}$.

For all $n, s \in \omega$ and all states $\hat{\nu}$, define $V_{n, s}=U_{n, s}^{a, g, B}, \hat{U}_{n, s}^{+}=\hat{V}_{n, s}^{+a, g, B}, \hat{U}_{n, s}=$ $\hat{V}_{n, s}^{a, g, B}, \hat{H}_{s}=H_{s}^{a, g, B}, \hat{C}_{s}=C_{s}^{a, g, B}, \hat{C}_{1, s}=C_{1, s}^{a, g, B}, \hat{C}_{2, s}=C_{2, s}^{a, g, B}, \hat{D}_{s}=D_{s}^{a, g, B}$, $\hat{B}_{\hat{\nu}, s}=B_{\hat{\nu}, s}^{a, g, B}, \hat{P}_{s}=P_{s}^{a, g, B}, \hat{Q}_{s}=Q_{s}^{a, g, B}, \hat{M}_{s}=M_{s}^{a, g, B}, \hat{\mathcal{M}}_{s}=\mathcal{M}_{s}^{a, g, B}, \hat{\mathcal{P}}_{s}=$ $\mathcal{P}_{s}^{a, g, B}{ }^{1} \hat{\mathcal{R}}_{s}={ }^{1} \mathcal{R}_{s}^{a, g, B}, \hat{\mathcal{I}}_{s}=\mathcal{I}_{s}^{a, g, B}, \hat{\mathcal{L}}_{\hat{\nu}, s}=\mathcal{L}_{\hat{\nu}, s}^{a, g, B}, \hat{\nu}_{s}=\nu_{s}^{a, g, B}, \hat{q}_{s}=q_{s}^{a, g, B}, \hat{d}_{s}=$ $d_{s}^{a, g, B}, \mathcal{S}_{s}(\hat{C})=\mathcal{S}_{s}^{a, g, B}(C), \mathcal{S}_{s}\left(\hat{C}_{1}\right)=\mathcal{S}_{s}^{a, g, B}\left(C_{1}\right), \mathcal{S}_{s}\left(\hat{C}_{2}\right)=\mathcal{S}_{s}^{a, g, B}\left(C_{2}\right), \mathcal{S}_{s}(\hat{D})=$ $\mathcal{S}_{s}^{a, g, B}(D), \mathcal{S}_{s}(\hat{P})=\mathcal{S}_{s}^{a, g, B}(P), \mathcal{S}_{s}(\hat{Q})=\mathcal{S}_{s}^{a, g, B}(Q), \hat{w}_{s}=w_{s}^{a, g, B}$, and $\hat{n}_{s}=n_{s}^{a, g, B}$. 
For all $i$, let $U_{i}=\bigcup_{s} U_{i, s}, \hat{V}_{i}=\bigcup_{s} \hat{V}_{i, s}, V_{i}=\bigcup_{s} V_{i, s}$, and $\hat{U}_{i}=\bigcup_{s} \hat{U}_{i, s}$. The reader can easily verify from the fact that $F$ and $G$ are recursive functionals that there are recursive functions $h, \hat{h}: \omega \rightarrow \omega$ such that $\hat{V}_{i}=W_{h(i)}^{A}$ and $\hat{U}_{i}=W_{\hat{h}(i)}^{B}$ for all $i$. If $e, x, \hat{x} \in \omega$, put $\nu(e, x)=\nu\left(e, x ;\left(U_{i}\right)_{i \in \omega},\left(\hat{V}_{i}\right)_{i \in \omega}\right)$ and $\hat{\nu}(e, \hat{x})=\nu\left(e, \hat{x} ;\left(V_{i}\right)_{i \in \omega},\left(\hat{U}_{i}\right)_{i \in \omega}\right)$. We say that $x$ has state $\nu$ at stage $s$ if $x \in M_{s}$ and $\nu \preceq \nu_{s}(x, x)$. We say that $x$ has final state $\nu$ if $\nu \preceq \nu(x, x)$.

For each $X$ one of the symbols $C, C_{1}, C_{2}, D, P, Q$, and each $s \in \omega$, choose a sequence $\mathcal{S}^{s}(X)$ listing the elements of $\mathcal{S}_{s}(X)$, in some arbitrary order. Let $\mathcal{S}(X)$ be the concatenation of the sequences $\mathcal{S}^{s}(X)$ for $s \in \omega$. If $\mathcal{L}$ is a sequence of elements of $S \times \omega$ and if $\nu \in S$, then we say that $n \in \omega$ is an occurrence of $\nu$ on $\mathcal{L}$ if for some $y \in \omega, \mathcal{L}(n)=(\nu, y)$.

We will show in the next section that (1)-(3) of Section 3 hold, from which it will follow, by (4) of Section 3 , that $\left(\mathcal{E}^{A}\right)^{*}$ and $\left(\mathcal{E}^{B}\right)^{*}$ are effectively isomorphic via an isomorphism $\Phi$ such that for all $i, \Phi\left(U_{i}^{*}\right)=\hat{U}_{i}^{*}$ and $\Phi\left(\hat{V}_{i}^{*}\right)=V_{i}^{*}$.

\section{Proof of the hard direction of the Main Theorem}

The proof is similar to the proof in Soare [9]. Each of the following lemmas has a dual lemma, obtained by exchanging the symbols $C$ and $\hat{C}$, and so on. In Lemma 14, both versions are proved simultaneously. Otherwise, the statement of the dual lemma is omitted.

Lemma 1. Every state which occurs infinitely often in $\mathcal{S}(C)$ also occurs infinitely often in each of $\mathcal{S}\left(C_{1}\right), \mathcal{S}\left(C_{2}\right)$, and $\mathcal{S}(D)$.

Proof. Let ${ }^{1} \mathcal{R}=\bigcup_{s}{ }^{1} \mathcal{R}_{s}$. Assume $\nu$ occurs infinitely often in $\mathcal{S}(C)$. To show that $\nu$ occurs infinitely often in $\mathcal{S}\left(C_{i}\right)$ for each $i \in\{1,2\}$, it suffices (cf. Rule $R_{1}$ ) to show that for each $i \in\{1,2\}$ and for each $n \in \omega$, there is an $m \geq n$ such that ${ }^{0} \mathcal{R}(m)=(\nu, i)$ and $m \in{ }^{1} \mathcal{R}$. So let $i \in\{1,2\}$ and $n \in \omega$. Choose $m \geq n$ such that $(\nu, i)={ }^{0} \mathcal{R}(m)$. Choose $s$ such that for all $k \leq m$ such that $k \in{ }^{1} \mathcal{R}, k \in{ }^{1} \mathcal{R}_{s}$. Since $\nu$ occurs infinitely often in $\mathcal{S}(C)$, there is a $t \geq s$ and a $y \in \omega$ such that $(\nu, y) \in \mathcal{S}_{t}(C)$. So $m \in{ }^{1} \mathcal{R}$ (for otherwise (cf. Rule $R_{1}$ ) there would be a $k \leq m$ such that $\left.k \in{ }^{1} \mathcal{R}_{t+1}-{ }^{1} \mathcal{R}_{t}\right)$. This completes the proof that $\nu$ occurs infinitely often in $\mathcal{S}\left(C_{1}\right)$ and in $\mathcal{S}\left(C_{2}\right)$.

To see that $\nu$ occurs infinitely often in $\mathcal{S}(D)$, notice that for all $s$, if $y \in C_{2, s}$ then $y \in D_{s+1}$ and $\nu_{s}(y, y)=\nu_{s+1}(y, y)$. Therefore since $\nu$ occurs infinitely often in $\mathcal{S}\left(C_{2}\right), \nu$ also occurs infinitely often in $\mathcal{S}(D)$.

If $\left(\mathcal{L}_{s}\right)_{s \in \omega}$ is a sequence of sets, then we let $\mathcal{L}_{\omega}=\left\{x \mid x \in \mathcal{L}_{s}\right.$ for all but finitely many $s \in \omega\}$. In particular, we use this notation for $\mathcal{M}_{\omega}, \mathcal{P}_{\omega}, P_{\omega}, Q_{\omega}$, and $B_{\nu, \omega}$.

Lemma 2. (i) If $y \in Q_{s}$ for infinitely many $s$, then $y \in Q_{\omega}$.

(ii) Suppose $y \in Q_{\omega}$. Then there exists a unique state $\nu$ such that $\lim _{s} q_{s}(\nu)$ exists and equals $y$. This state $\nu$ satisfies $\nu \preceq \lim _{s} \nu_{s}(y, y)$.

(iii) For every $\nu$, if $\lim _{s} q_{s}(\nu)$ exists then $\lim _{s} q_{s}(\mu)$ exists for every $\mu \preceq \nu$.

Proof. (i) It is easy to see that if $y \in Q_{s}-Q_{s+1}$, then $\nu_{s}(y, y) \subsetneq \nu_{s+1}(y, y)$. But there are only finitely many states of length $y$, so $\nu_{s}(y, y) \subsetneq \nu_{s+1}(y, y)$ for only finitely many $s$.

We prove (ii) by induction on $y$. Assume that (ii) is true for all $z<y$, and that $y \in Q_{\omega}$. Choose $s_{0}$ so that (1) for all $z \leq y$ and for all $s \geq s_{0}, z \in Q_{s}$ iff $z \in Q_{s_{0}}$; 
(2) for all $z<y$, all $s \geq s_{0}$, and all states $\mu$, if $q_{s_{0}}(\mu) \downarrow$ and $q_{s_{0}}(\mu)=z$, then $q_{s}(\mu) \downarrow$ and $q_{s}(\mu)=z$; and $(3)$ for all $s \geq s_{0}, \nu_{s}(y, y)=\nu_{s_{0}}(y, y)$. Since $y \in Q_{s_{0}}, y=q_{s_{0}}(\nu)$ for some state $\nu$. By the definition of $q$, for all $\mu \prec \nu, q_{s_{0}}(\mu) \downarrow$ and $q_{s_{0}}(\mu)<y$. So by (2), for all $s \geq s_{0}$ and all $\mu \prec \nu, q_{s}(\mu) \downarrow$ and $q_{s}(\mu)=q_{s_{0}}(\mu)$. Thus (using (3)), for any $s \geq s_{0}, q_{s}(\nu) \downarrow$ and $q_{s}(\nu) \leq y$. Suppose $q_{s}(\nu)=z<y$ for some $s>s_{0}$. So by $(2), q_{s}(\nu)=q_{s_{0}}(\nu)$. But $q_{s_{0}}(\nu)=y$, a contradiction. The rest of the statement of (ii) follows directly from the definition of $q$.

(iii) Suppose $\lim _{s} q_{s}(\nu)$ exists and equals $y$. Choose $s_{0}$ large enough so that (1) for all $z<y$ and for all $s \geq s_{0}, z \in Q_{s}$ iff $z \in Q_{s_{0}}$, (2) for all $z<y$, all $s \geq s_{0}$, and all states $\mu$, if $q_{s_{0}}(\mu) \downarrow$ and $q_{s_{0}}(\mu)=z$ then $q_{s}(\mu) \downarrow$ and $q_{s}(\mu)=z$. (The existence of an $s_{0}$ for (2) follows from part (ii) of the lemma.) But by definition of $q$, for any $\mu \prec \nu$, there is a $z<y$ such that $q_{s_{0}}(\mu)=z$. So by $(2)$, for all $s \geq s_{0}, q_{s}(\mu) \downarrow$ and $q_{s}(\mu)=z$.

Lemma 3. Fix a state $\hat{\nu}$. If for infinitely many $s \in \omega$ there is some $\hat{\mu} \succcurlyeq \hat{\nu}$ such that $\hat{\mu} \in \mathcal{M}_{s+1}-\mathcal{M}_{s}$, then $\hat{\nu}$ occurs in $\mathcal{S}(\hat{D})$ infinitely often.

Proof. Let $T$ be the set of $s$ such that there exists a $\hat{\mu} \succcurlyeq \hat{\nu}$ such that $\hat{\mu} \in \mathcal{M}_{s+1}-\mathcal{M}_{s}$. For each $s \in T$, let $\hat{\mu}_{s}$ be a state $\succcurlyeq \hat{\nu}$ such that $\hat{\mu}_{s} \in \mathcal{M}_{s+1}-\mathcal{M}_{s}$, and define $t_{s}$ as follows (cf. the definition of $\mathcal{M}$ ): let $t_{s}$ be the largest $t<s+1$ such that $\hat{\mu}_{s} \in \mathcal{M}_{t}$, if such a $t$ exists, and let $t_{s}=\left|\hat{\mu}_{s}\right|$ otherwise. If $\sup _{s \in T}\left|\hat{\mu}_{s}\right|=\infty$, then $\sup _{s \in T} t_{s}=\infty$, as the reader can easily verify. Otherwise for some fixed $\hat{\pi}, \hat{\pi}=\hat{\mu}_{s}$ for infinitely many $s \in T$. Thus since $\hat{\pi} \in \mathcal{M}_{u}$ for infinitely many $u$, we again see that $\sup _{s \in T} t_{s}=\infty$. Since $\hat{\nu} \preceq \hat{\mu}_{s}$ for each $s, \alpha(\hat{\nu})$ is verified infinitely often (by the definition of $\mathcal{M})$, and thus $\left\{\hat{y} \mid(\exists s)\left[(\hat{\nu}, \hat{y}) \in \mathcal{S}_{s}(\hat{D})\right]\right\}$ is infinite.

Lemma 4. Suppose $\lim _{s} \hat{q}_{s}\left(\mu^{*}\right)$ exists and yet $B_{\mu, s}=\emptyset$ for infinitely many s. Then for all $\nu \succcurlyeq \mu$ such that $\nu$ occurs in $\mathcal{S}(D)$ infinitely often there is a state $\nu^{\prime}>_{*} \nu$ such that $\nu^{\prime}$ occurs in $\mathcal{S}(D)$ infinitely often.

Proof. Let $\mu$ and $\nu$ be as in the statement of the lemma. By the dual of Lemma 2(iii), we may assume without loss of generality that for all $\pi \prec \mu, B_{\pi, s}=\emptyset$ for only finitely many $s$; otherwise we can shorten $\mu$. So, as is easily seen, for each $\pi \prec \mu$, $\bigcup_{s} \mathcal{L}_{\pi, s+1}$ is finite (cf. Rule $R_{2}$, Step 4). Using the facts that $\nu$ occurs in $\mathcal{S}(D)$ infinitely often and that $B_{\mu, s}=\emptyset$ for infinitely many $s$, the reader can easily verify that for infinitely many $s$, there is a $y \in B_{\mu, s}$ which is in state $\nu$ at stage $s$. (Hint: by Rule $R_{2}$, Step $4, \nu$ will occur unmarked on $\mathcal{L}_{\mu, s+1}$ for infinitely many $s$, and by Rule $R_{2}$, Step 5 , each unmarked occurrence of $\nu$ in $\mathcal{L}_{\mu, s+1}$ will be marked at some stage $t>s$.) Let $T$ be the set of pairs $(y, s)$ such that $y \in B_{\mu, s}$ and $y$ is in state $\nu$ at stage $s$. For each pair $(y, s) \in T$, let $t(y, s)$ be the least $t \geq s$ such that $y \in B_{\mu, t}-B_{\mu, t+1}$. $B_{\mu, t(y, s)} \neq B_{\mu, t(y, s)+1}$, so $B_{\mu, t(y, s)+1}$ is defined either by Rule $R_{2}$ or by Case $3 \mathrm{~B} 2$. It is easy to see that if there were infinitely many $(y, s) \in T$ such that $B_{\mu, t(y, s)+1}$ is defined by Rule $R_{2}$, then $\gamma\left(\mu^{*}\right)$ would be verified infinitely often and therefore $\left\{s \mid \hat{q}_{s}\left(\mu^{*}\right) \uparrow \vee(\exists t>s)\left(\hat{q}_{t}\left(\mu^{*}\right) \uparrow \vee \hat{q}_{t}\left(\mu^{*}\right) \neq \hat{q}_{s}\left(\mu^{*}\right)\right)\right\}$ would be infinite. But $\lim _{s} \hat{q}_{s}\left(\mu^{*}\right)$ exists.

Let $\hat{T}$ be the set of all pairs $(y, s) \in T$ such that $B_{\mu, t(y, s)+1}$ is defined by Case 3B2. Let $e=|\nu|$. We will show that for each $(y, s) \in \hat{T}, \nu_{t(y, s)+1}(e, y)>_{*} \nu_{s}(e, y)=\nu$. Given this, if $u>t(y, s)$ is least such that $y \in C_{u}$, then $\nu_{u}(e, y)=\nu_{t(y, s)+1}(e, y)$ (i.e., $y$ retains the same state while it is in hole $H$ ). So some $\nu^{\prime}>_{*} \nu$ occurs infinitely often in $\mathcal{S}(C)$, and thus by Lemma $1, \nu^{\prime}$ occurs infinitely often in $\mathcal{S}(D)$. 
Now suppose $(y, s) \in \hat{T}$. It is easily seen that $\nu_{t(y, s)+1}(y, y) \geq_{*} \nu_{s}(y, y)$ (i.e., $y$ enters no set $\hat{V}_{i}$ while $y$ is in $P$ ). However, since $y$ leaves $B_{\mu}$ through Case 3B2, we see that $y \in U_{i, t(y, s)+1}-U_{i, t(y, s)}$ for some $i \leq|\mu| \leq e$. Thus $\nu_{t(y, s)+1}(e, y)>_{*}$ $\nu_{s}(e, y)$.

Lemma 5. Assume $\hat{\nu}$ is a state such that there are only finitely many occurrences of states $\hat{\nu}^{\prime}>_{*} \hat{\nu}$ in $\mathcal{S}(\hat{D})$. Then there are only finitely many s such that $\hat{\nu} \in \mathcal{M}_{s}$ and Condition (b) holds of $\hat{\nu}$ at stage $s$.

Proof. Let $\hat{\nu}$ be as in the statement of the lemma. Suppose by way of contradiction that there are infinitely many $s$ such that $\hat{\nu} \in \mathcal{M}_{s}$ and Condition (b) holds of $\hat{\nu}$ at stage $s$. So for infinitely many $s, \hat{\nu} \in \mathcal{M}_{s}-\mathcal{M}_{s+1}$. Thus for infinitely many $s$, $\hat{\nu} \in \mathcal{M}_{s+1}-\mathcal{M}_{s}$. So by Lemma $3, \hat{\nu}$ occurs in $\mathcal{S}(\hat{D})$ infinitely often. Using the fact that there are infinitely many $s$ such that Condition (b) holds of $\hat{\nu}$ at stage $s$, the reader can easily verify that there must be a state $\hat{\mu} \preceq \hat{\nu}$ such that (1) $q_{v}\left(\hat{\mu}^{*}\right)$ has a constant value for all $v$ with $|\hat{\nu}| \leq v$, and $(2) \beta(\hat{\mu})$ is verified infinitely often. By (2), $\left\{s \mid \hat{B}_{\hat{\mu}, s}=\emptyset\right\}$ is infinite. By the dual of Lemma 4, there is some state $\hat{\nu}^{\prime}>_{*} \hat{\nu}$ which occurs infinitely often in $\mathcal{S}(\hat{D})$. This is a contradiction.

Lemma 6. Fix e. Assume that for all states $\hat{\nu}$ of length $\leq e$, there are only finitely many such that $\hat{\nu} \in \mathcal{M}_{s}$ and Condition (a) holds of $\hat{\nu}$ at stage s. Then for all states $\nu$ and $\hat{\nu}$ of length $e$ :

(i) If $\hat{\nu}$ occurs in $\mathcal{S}(\hat{D})$ infinitely often, then some $\hat{\mu} \geq_{*} \hat{\nu}$ is in $\mathcal{M}_{\omega}$.

(ii) If some $\hat{\nu}_{*} \| \nu$ occurs infinitely often in $\mathcal{S}(\hat{D})$, then $\nu \in \mathcal{P}_{\omega}$.

(iii) If $\nu \in \mathcal{P}_{s}$ for infinitely many $s$, then $\nu \in \mathcal{P}_{\omega}$.

Proof. (i) Suppose $\hat{\nu}$ occurs in $\mathcal{S}(\hat{D})$ infinitely often. Choose $\hat{\mu}$ such that $\hat{\mu} \geq_{*} \hat{\nu}$ and $\hat{\mu}$ occurs in $\mathcal{S}(\hat{D})$ infinitely often, but for no $\hat{\mu}^{\prime}>_{*} \hat{\mu}$ does $\hat{\mu}^{\prime}$ occur in $\mathcal{S}(\hat{D})$ infinitely often. So by Lemma 5 , there are only finitely many such that $\hat{\mu} \in \mathcal{M}_{s}$ and Condition (b) holds of $\hat{\mu}$ at stage $s$. But by assumption, there are only finitely many $s$ such that $\hat{\mu} \in \mathcal{M}_{s}$ and Condition (a) holds of $\hat{\mu}$ at stage $s$. Therefore there are only finitely many $s$ such that $\hat{\mu} \in \mathcal{M}_{s}-\mathcal{M}_{s+1}$. Thus, since $\hat{\mu}$ occurs in $\mathcal{S}(\hat{D})$ infinitely often, $\hat{\mu} \in \mathcal{M}_{\omega}$.

(ii) is immediate from (i), from Proposition 3.1, and from the definition of $\mathcal{P}_{\omega}$.

(iii) Suppose $\nu$ is of length $e$ and $\nu \in \mathcal{P}_{s}$ for infinitely many $s$. For each $s$ such that $\nu \in \mathcal{P}_{s}$ there is a $\hat{\nu} \in \mathcal{M}_{s}$ such that $\hat{\nu}{ }_{*} \| \nu$. Choose $\hat{\nu}{ }_{*} \| \nu$ such that $\hat{\nu} \in \mathcal{M}_{s}$ for infinitely many $s$. If $\hat{\nu} \in \mathcal{M}_{\omega}$, then $\nu \in \mathcal{P}_{\omega}$. Otherwise, $\hat{\nu} \in \mathcal{M}_{s+1}-\mathcal{M}_{s}$ for infinitely many $s$. So by Lemma $3, \hat{\nu}$ occurs in $\mathcal{S}(\hat{D})$ infinitely often. Apply (i) to find a $\hat{\mu} \geq_{*} \hat{\nu}$ such that $\hat{\mu} \in \mathcal{M}_{\omega}$. But $\hat{\nu}{ }_{*} \| \nu$, so by Proposition 3.1, $\hat{\mu} * \| \nu$. Thus $\nu \in \mathcal{P}_{\omega}$.

Lemma 7. (1) Every $x \in \omega$ is either in $P_{\omega}$ or in $Q_{\omega}$. (2) For each $\nu, B_{\nu, \omega}$ is finite. (3) If $B_{\nu, s} \neq \emptyset$ for all but finitely many $s$, then $B_{\nu, \omega} \neq \emptyset$. Finally, (4) each element of $B_{\nu, \omega}$ has final state $\nu$.

Proof. We prove (1) by proving that (1a) $\bigcup_{s} M_{s} \subseteq P_{\omega} \cup Q_{\omega}$, and (1b) $\bigcup_{s} M_{s}=\omega$.

(1a): We prove by induction on $x$ that if $x \in \cup_{s} M_{s}$, then $x \in P_{\omega} \cup Q_{\omega}$. So suppose that $x \in \bigcup_{s} M_{s}$ and that for all $y<x$, if $y \in \bigcup_{s} M_{s}$, then $y \in P_{\omega} \cup Q_{\omega}$. Observe that $M_{s} \subseteq M_{s+1}$ for all $s$. Choose $s$ such that (i) $x \in M_{s}$; and for all $y<x$, either (ii) for all $t \geq s, y \in P_{t}$ or (iii) for all $t \geq s, y \in Q_{t}$. Notice that for all $t \geq s$, (iv) if $x \in H_{t}$ then $x \in C_{t+1}$, (v) if $x \in C_{t}$ then $x \in C_{1, t+1} \cup C_{2, t+1}$, (vi) 
if $x \in C_{1, t} \cup C_{2, t}$ then $x \in D_{t+1}$, and (vii) if $x \in D_{t}$ then $x \in P_{t+1} \cup Q_{t+1}$. Thus for infinitely many $t, x \in P_{t} \cup Q_{t}$. But for all $t$, if $x \in P_{t}-P_{t+1}$ or $x \in Q_{t}-Q_{t+1}$, then $\nu_{t+1}(x, x) \supsetneq \nu_{t}(x, x)$. But since there are only finitely many states of length $x, \nu_{t+1}(x, x) \supsetneq \nu_{t}(x, x)$ for only finitely many $t$. Thus $x \in P_{\omega} \cup Q_{\omega}$, completing the induction step and thus the proof of (1a).

(1b): It is easy to see by induction on $s$ that for all $s, M_{s}=\{x \mid(\exists t<$ $\left.\left.n_{s}\right)\left[g^{A}(t)=(0, x)\right]\right\}$. Thus to prove (1b), it suffices to show that $\lim _{s} n_{s}=\infty$. So suppose $\lim _{s} n_{s}<\infty$. Choose $s$ such that $n_{t}=n_{t+1}$ for all $t \geq s$. Thus $\bigcup_{t} M_{t}$ equals $M_{s}$ and is therefore finite. Using (1a), choose $s^{\prime}>0$ such that $s^{\prime} \geq s$ and such that for all $x \in \bigcup_{t} M_{t}$, either (i) for all $t \geq s^{\prime}, x \in P_{t}$, or (ii) for all $t \geq s^{\prime}$, $x \in Q_{t}$. But then $s^{\prime}>0$ and $H_{s^{\prime}} \cup C_{s^{\prime}} \cup C_{1, s^{\prime}} \cup C_{2, s^{\prime}} \cup D_{s^{\prime}}=\emptyset$. Consequently, $n_{s^{\prime}+1}=n_{s^{\prime}}+1$ by Case 3 of Section 4.2.1, a contradiction. This completes the proof of (1b) and thus the proof of (1).

(2): If $B_{\nu, \omega} \neq \emptyset$, then $B_{\nu, s} \neq \emptyset$ for all sufficiently large $s$. So $\bigcup_{s}{ }^{+} \mathcal{L}_{\nu, s}$ is finite (cf. Rule $R_{2}$, Step 4). So $\bigcup_{s} B_{\nu, s}$ is finite (cf. Rule $R_{2}$, Step 5).

(3): If $B_{\nu, s} \neq \emptyset$ for cofinitely many $s$, then, by the previous argument, $\bigcup_{s} B_{\nu, s}$ is finite. Let $s_{0}$ be large enough so that for all $x$ in $\bigcup_{s} B_{\nu, s}$, either $x \in P_{s}$ for all $s \geq s_{0}$ or $x \in Q_{s}$ for all $s \geq s_{0}$. We claim $B_{\nu, s}=B_{\nu, s_{0}}$ for all $s \geq s_{0}$. For consider $y \in \bigcup_{s} B_{\nu, s}$. By definition of $s_{0}$, if $y \in B_{\mu, s_{0}}$ for some state $\mu$, then for no $s>s_{0}$ can we have $y \notin B_{\mu, s}$ (i.e., if $y$ leaves $B_{\mu}$, then $y$ must enter either $Q$ or $H$, contradicting the choice of $s_{0}$ ). Thus if $y \in B_{\nu, s_{0}}$, then $y \in B_{\nu, s}$ for all $s \geq s_{0}$. On the other hand, suppose $y \notin B_{\nu, s_{0}}$. Then since $y \in P_{s_{0}}$ or $y \in Q_{s_{0}}$, either $y \in B_{\mu, s_{0}}$ for some $\mu \neq \nu$ or $y \in Q_{s_{0}}$. In either case, $y \notin B_{\nu, s}$ for all $s \geq s_{0}$. Thus $B_{\nu, s}=B_{\nu, s_{0}}$ for all $s \geq s_{0}$. Since by assumption $B_{\nu, s} \neq \emptyset$ for some $s \geq s_{0}$, we have $B_{\nu, \omega} \neq \emptyset$.

(4): Finally, for all $x \in B_{\nu, s+1}, \nu_{s+1}(|\nu|, x)=\nu_{s}(|\nu|, x)$. So if $x \in B_{\nu, \omega}, x$ has final state $\nu$.

Lemma 8. Fix $\nu$ and $X$. For all but finitely many $x$, if there is an occurrence of $(\nu, x)$ in $\mathcal{S}(X)$, then for some $\nu^{\prime} \geq \nu$ there is an occurrence of $\left(\nu^{\prime}, x\right)$ in $\mathcal{S}(C)$.

Proof. Suppose $(\nu, x) \in \mathcal{S}_{s}(X)$. So $x \in X_{s}$ and $\nu \preceq \nu_{s}(x, x)$. If $X=C$ the lemma is trivial. If $X=C_{1}$ or $C_{2}$, then $x \in C_{s-1}$ and $\nu \preceq \nu_{s-1}(x, x)\left(=\nu_{s}(x, x)\right)$. Otherwise, let $\nu=(e, \sigma, \tau)$. Let $t$ be the largest $t<s$ such that $x \in C_{t}$. Let $\nu^{\prime}=\nu_{t}(e, x)=\left(e, \sigma^{\prime}, \tau^{\prime}\right)$. If $X=D$ or $X=Q$ then $\sigma=\sigma^{\prime}$, so $\nu^{\prime} \geq \nu$. Suppose now that $X=P$. Let $\mu$ be such that $x \in B_{\mu, s}$. If $|\mu| \geq e$, then $\sigma=\sigma^{\prime}$ (or else $x \notin P_{s}$ ), and $\nu^{\prime} \geq \nu$ as before. So we may assume that $|\mu|<e$. If $x \notin B_{\mu, \omega}$, let $u$ be the least $u \geq s$ such that $x \in B_{\mu, u}-B_{\mu, u+1}$. $B_{\mu, u+1}$ is defined either by Rule $R_{2}$ or by Case 3B2. So either $x \in Q_{u+1}-Q_{u}$ (in which case we have $\sigma=\sigma^{\prime}$, since $\left.\nu \preceq \nu_{s}(x, x)\right)$ or $x \in H_{u+1}-H_{u}$. In the former case, $\nu^{\prime} \geq \nu$, so we are done. In the latter case, $\nu_{u+1}(e, x)=\left(e, \sigma^{\prime \prime}, \tau\right)$ for some $\sigma^{\prime \prime} \supseteq \sigma$. So if $v$ is the smallest $v>u$ such that $x \in C_{v}$, then $\nu_{v}(e, x)=\nu_{u+1}(e, x) \geq \nu$. The lemma now follows from the facts that $|\mu|<e$ and that by Lemma 7 , for each state $\mu, B_{\mu, \omega}$ is finite.

Lemma 9. For each $x$ and $X$, there are only finitely many s such that for some $\nu,(\nu, x) \in \mathcal{S}_{s}(X)$.

Proof. By Lemma 7, we choose $s_{0}$ large enough so that either (1) for all $s \geq s_{0}$, $x \in P_{s}$, or (2) for all $s \geq s_{0}, x \in Q_{s}$. So if $X=C, C_{1}, C_{2}$, or $D$, then there are no $s \geq s_{0}$ and state $\nu$ such that $(\nu, x) \in \mathcal{S}_{s}(X)$. If $X=P$ or $X=Q$, choose $s_{1}>s_{0}$ such that $\nu_{s}(x, x)=\nu_{s_{1}}(x, x)$ for all $s \geq s_{1}$. So $(\nu, x) \notin \mathcal{S}_{s}(X)$ for any $s \geq s_{1}$ and state $\nu$. 
For each state $\hat{\mu}$, we let $\hat{\mathcal{H}}_{\hat{\mu}}=\left\{s \mid(\exists \hat{y})(\exists X)\left[\hat{\nu}_{s}(|\hat{\mu}|, \hat{y}) \downarrow \wedge\left(\hat{\nu}_{s}(|\hat{\mu}|, \hat{y}), \hat{y}\right) \in \mathcal{S}_{s}(\hat{X}) \wedge\right.\right.$ $\left.\left.\hat{\nu}_{s}(|\hat{\mu}|, \hat{y}) \notin \hat{\mathcal{P}}_{s} \wedge(\exists t \leq s)\left[\hat{\nu}_{t}(|\hat{\mu}|, \hat{y}) \downarrow \wedge \hat{\mu}=\hat{\nu}_{t}(|\hat{\mu}|, \hat{y})\right]\right]\right\}$ (cf. the definition of $\left.\delta(\hat{\mu})\right)$. Roughly speaking, $\hat{\mathcal{H}}_{\hat{\nu}}$ is infinite exactly if there are infinitely many elements $\hat{y}$ which are at some stage in state $\hat{\nu}$ and at some later stage "cause" a failure of Lemma 14 (2) for a state of the same length as $\hat{\nu}$. Lemma 14 will show that the sets $\hat{\mathcal{H}}_{\hat{\nu}}$ are actually finite.

Lemma 10. Fix a state $\hat{\nu}$. If $\hat{\mathcal{H}}_{\hat{\nu}}$ is infinite and some $\nu \| \hat{\nu}$ occurs infinitely often in $\mathcal{S}(C)$, then some $\nu^{\prime}{ }_{*} \| \hat{\nu}$ occurs infinitely often in $\mathcal{S}(D)$.

Proof. Fix $\hat{\nu}$ as in the statement of the lemma. Fix $\nu \| \hat{\nu}$ such that $\nu$ occurs infinitely often in $\mathcal{S}(C)$. Since $\hat{\mathcal{H}}_{\hat{\nu}}$ is infinite, $\delta(\hat{\nu})$ is verified at infinitely many stages $s$. Also, since $\hat{\mathcal{H}}_{\hat{\nu}}$ is infinite and by the dual of Lemma $9,\{s \mid(\exists \hat{y})(\exists X)[(\hat{\mu}, \hat{y}) \in$ $\left.\left.\mathcal{S}_{s}(\hat{X})\right]\right\}$ is certainly infinite for each $\hat{\mu} \preceq \hat{\nu}$. Thus for each $\hat{\mu} \preceq \hat{\nu}, \epsilon(\hat{\mu})$ is verified at infinitely many stages $s$. By Lemma 1 , since $\nu$ occurs infinitely often in $\mathcal{S}(C)$, $\nu$ also occurs infinitely often in $\mathcal{S}\left(C_{2}\right)$. Consider an unmarked occurrence of $\hat{\nu}$ on $\left({ }^{+} \mathcal{I}_{s},{ }^{-} \mathcal{I}_{s-1}\right)$. By Rule $R_{3}$, Step 3, each such occurrence is marked on some $\mathcal{I}_{t}$ for $t>s$. Suppose that $t>s$ is least. By the definition of Rule $R_{3}$, Step 3, then there is an $x_{t} \in \omega$ such that $x_{t} \in D_{t}, \nu_{t-1}\left(|\nu|, x_{t}\right) \| \hat{\nu}$ and $\nu_{t}\left(|\nu|, x_{t}\right) * \| \hat{\nu}$. Now, by Rule $R_{3}$, Step 2 , and the fact that $\delta(\hat{\nu})$ and $\epsilon(\hat{\mu})$ for all $\hat{\mu} \preceq \hat{\nu}$ are verified for infinitely many stages, $\hat{\nu}$ occurs unmarked on $\left({ }^{+} \mathcal{I}_{s},{ }^{-} \mathcal{I}_{s-1}\right)$ for infinitely many $s$. Thus for infinitely many $t$, there is an $x_{t} \in \omega$ such that $x_{t} \in D_{t}$ and $\nu_{t}\left(|\nu|, x_{t}\right) * \| \hat{\nu}$. Choose $\nu^{\prime}$ which is $\nu_{t}\left(|\nu|, x_{t}\right)$ for infinitely many such $x_{t}$. So $\nu_{*}^{\prime} \| \hat{\nu}$ and $\nu^{\prime}$ occurs infinitely often in $\mathcal{S}(D)$.

Let $\mathcal{I}=\bigcup_{s}{ }^{+} \mathcal{I}_{s}$ (i.e., the set-theoretic union of functions).

Lemma 11. Suppose that there are infinitely many $n$ such that $\mathcal{I}(n) \succcurlyeq \hat{\nu}$. Then $(\exists X)[\hat{\nu}$ occurs infinitely often in $\mathcal{S}(\hat{X})]$.

Proof. The proof is very similar to the proof of Lemma 3. Let $T$ be an infinite subset of $\omega$ such that for all $n \in T, \mathcal{I}(n) \succcurlyeq \hat{\nu}$. For each $n \in T$, let $u_{n}$ be such that $n \in \operatorname{dom} \mathcal{I}_{u_{n}+1}-\operatorname{dom} \mathcal{I}_{u_{n}}$, and let $t_{n}=w_{u_{n}+1}(\mathcal{I}(n))$ (cf. Rule $R_{3}$, Step 1). If $\sup _{n \in T}|\mathcal{I}(n)|=\infty$, it is easy to see that $\sup _{n \in T} t_{n}=\infty$. Otherwise for some fixed $\hat{\mu}, \mathcal{I}(n)=\hat{\mu} \succcurlyeq \hat{\nu}$ for infinitely many $n \in T$. Since $\hat{\mu}$ occurs in $\mathcal{I}$ infinitely often, $\hat{\mu}$ occurs unmarked on $\mathcal{I}_{t}$ for infinitely many $t$, so again it is easy to see that $\sup _{n \in T} t_{n}=\infty$. Since $\hat{\nu} \preceq \mathcal{I}(n)$ for each $n$, the reader can easily see that $\epsilon(\hat{\nu})$ is verified infinitely often, and therefore $\left\{s \mid(\exists \hat{y})(\exists X)\left[(\hat{\nu}, \hat{y}) \in \mathcal{S}_{s}(\hat{X})\right]\right\}$ is infinite.

For each state $\mu$, we put $\mathcal{H}_{\mu}=\left\{s \mid(\exists y)(\exists X)\left[\nu_{s}(|\mu|, y) \downarrow \wedge\left(\nu_{s}(|\mu|, y), y\right) \in\right.\right.$ $\left.\left.\mathcal{S}_{s}(X) \wedge \nu_{s}(|\mu|, y) \notin \mathcal{P}_{s} \wedge(\exists t \leq s)\left[\nu_{t}(|\mu|, y) \downarrow \wedge \mu=\nu_{t}(|\mu|, y)\right]\right]\right\}$ (cf. the definition of $\hat{\mathcal{H}})$.

Lemma 12. Fix e. The following conditions (1)-(4) are equivalent:

(1) $(\forall \nu$ of length $<e)(\forall X)\left[\nu\right.$ occurs in $\mathcal{S}(X)$ infinitely often $\left.\Rightarrow \nu \in \mathcal{P}_{\omega}\right]$.

(2) For all states $\nu$ of length $<e$ there are only finitely many s such that

$$
(\exists X)(\exists y)\left[(\nu, y) \in \mathcal{S}_{s}(X) \wedge \nu \notin \mathcal{P}_{s}\right] .
$$

(I.e, for all states $\hat{\nu}$ of length $\leq e$ there are only finitely many stages s such that Condition (a) holds of $\hat{\nu}$ at stage s.)

(3) $\mathcal{H}_{\nu}$ is finite for all states $\nu$ of length $<e$. 
(4) For all but finitely many $x$ : for all $s$ such that $x \in M_{s}, d_{s}(x) \geq e-1$.

If, in addition to (1)-(4) $\nu_{0}$ is of length e and

(5) $\left(\forall \nu \subseteq \nu_{0}\right)(\forall X)\left[\nu\right.$ occurs in $\mathcal{S}(X)$ infinitely often $\left.\Rightarrow \nu \in \mathcal{P}_{\omega}\right]$

then

(6) for all but finitely many $x$ : for all $s$ such that $x \in M_{s}$ and such that $\nu_{s}(e, x) \subseteq$ $\nu_{0}, d_{s}(x) \geq e$.

Proof. (1) $\Rightarrow(2)$ and (1) $\Rightarrow(3)$ are clear.

$(1) \Rightarrow(4)$ : First, consider $x \geq e$. We claim that for all stages $s$ such that $x \in M_{s}$, there is an $X$ and a $t \geq s$ such that $\left(\nu_{s}(e-1, x), x\right) \in \mathcal{S}_{t}(X)$ (i.e., for every state $\nu$ occupied by $x$ while $x$ is in $M,(\nu, x)$ enters some $\mathcal{S}(X))$. First, if $s$ is least such that $x \in M_{s}$, then $x \in H_{s}$. For any $s$ such that $x \in H_{s}$, we have $x \in C_{t}$ for some $t>s$, and for $t$ least, $\left(\nu_{s}(e-1, x), x\right)=\left(\nu_{t}(e-1, x), x\right) \in \mathcal{S}_{t}(C)$. Also, for any $s$ such that $x \in M_{s-1}$ and $\nu_{s}(e-1, x) \neq \nu_{s-1}(e-1, x)$, either Step 3 of Rule $R_{3}$, or Case 3B, or Rule $R_{4}$ applies. If Step 3 of Rule $R_{3}$ applies, then $\left(\nu_{s}(e-1, x), x\right) \in \mathcal{S}_{s}(D)$. If Rule $R_{4}$ applies, then $\left(\nu_{s}(e-1, x), x\right) \in \mathcal{S}_{s}(Q)$. If Case 3B applies, then either $x \in H_{s}$, in which case we have seen that for $t>s$ least such that $x \in C_{t}$, then $\left(\nu_{s}(e-1, x), x\right) \in \mathcal{S}_{t}(C)$; or else $x \in Q_{s}$, in which case we have $\left(\nu_{s}(e-1, x), x\right) \in \mathcal{S}_{s}(Q)$.

Now assume (1) holds. Choose $s_{0}$ such that for all $\nu$ of length $e-1, \nu \in \mathcal{P}_{\omega}$ implies $\nu \in \mathcal{P}_{s}$ for all $s \geq s_{0}$. For each state $\nu$ of length $e-1$ and each $X$ such that $\nu$ does not occur infinitely often in $\mathcal{S}(X)$, let $F_{\nu, X}=\left\{x \mid(\exists s)\left[\left(\nu_{s}(e-1, x)=\nu\right.\right.\right.$ and $\left.\left.(\nu, x) \in \mathcal{S}_{s}(X)\right]\right\}$. Note that each $F_{\nu, x}$ is finite. Let $G=\bigcup_{\nu, X} F_{\nu, x}$. There are only finitely many $e-1$ states, so $G$ is finite. Now choose $x_{0}$ such that (a) $x_{0}>e-1$, (b) $x_{0}>\max G$, and (c) no $x \geq x_{0}$ is in $M_{s_{0}}$. So by (1), for each $x \geq x_{0}$ and each $s$ such that $x \in M_{s},\left(\nu_{s}(e-1, x), x\right) \in \mathcal{P}_{\omega}$, and thus since $s>s_{0}$, $\left(\nu_{s}(e-1, x), x\right) \in \mathcal{P}_{s}$. Thus by the definition of $d$, for all $s$ such that $x \in M_{s}$, $d_{s}(x) \geq e-1$.

(2) $\Rightarrow$ (1): If $\nu$ is of length $<e$ and $\nu \notin \mathcal{P}_{\omega}$, then by Lemma 6 , there is some $s_{0}$ such that for all $s \geq s_{0}, \nu \notin \mathcal{P}_{s}$. Thus by (2) again, $\nu$ cannot occur in $\mathcal{S}_{s}(X)$ infinitely often.

$(3) \Rightarrow(2)$ and $(4) \Rightarrow(2)$ are clear.

The proof that $(1) \wedge(5) \Rightarrow(6)$ is similar to the proof that $(1) \Rightarrow(4)$, and is left for the reader.

Showing that (1)-(4) hold for all $e$ is the main remaining obstacle in the proof. We will show this by induction on $e$ in Lemma 14. The next lemma plays a crucial part in this induction.

Lemma 13. Fix e. Assume

$$
(\forall \nu \text { of length }<e)(\forall X)\left[\nu \text { occurs in } \mathcal{S}(X) \text { infinitely often } \Rightarrow \nu \in \mathcal{P}_{\omega}\right]
$$

and

$$
(\forall \hat{\nu} \text { of length }<e)(\forall \hat{X})\left[\hat{\nu} \text { occurs in } \mathcal{S}(\hat{X}) \text { infinitely often } \Rightarrow \hat{\nu} \in \hat{\mathcal{P}}_{\omega}\right] .
$$

Then for all states $\nu$ of length e such that $\mathcal{H}_{\nu}$ is infinite, there is some $\hat{\nu} \| \nu$ which occurs infinitely often in $\mathcal{S}(\hat{C})$. 
Proof. First, a technical definition is useful. For each $e$ and for each pair of states $\mu$ and $\mu_{+}$of length $e$, we put

$$
\begin{aligned}
& \mathcal{H}_{\mu, \mu_{+}}=\{s \mid(\exists y)(\exists X)[\left(\mu_{+}, y\right) \in \mathcal{S}_{s}(X) \wedge \mu_{+} \notin \mathcal{P}_{s} \\
&\left.\left.\wedge(\exists t \leq s)\left[\nu_{t}(e, y) \downarrow \wedge \mu=\nu_{t}(e, y)\right]\right]\right\} .
\end{aligned}
$$

The lemma will now be proved by $\subseteq$-induction on $\nu$. Assume the claim is true for all $\nu_{-} \subset \nu$. We prove by $\subseteq$-induction on $\nu_{+}$that if $\mathcal{H}_{\nu_{\nu} \nu_{+}}$is infinite then there is some $\hat{\nu} \| \nu$ which occurs infinitely often in $\mathcal{S}(\hat{C})$. So assume that $\mathcal{H}_{\nu, \nu_{+}}$is infinite and that for all $\nu_{+}^{\prime} \subset \nu_{+}$, if $\mathcal{H}_{\nu, \nu_{+}^{\prime}}$ is infinite then there is some $\hat{\nu} \| \nu$ which occurs infinitely often in $\mathcal{S}(\hat{C})$. Let $\nu=(e, \sigma, \tau)$. Choose $y_{j}, s_{j}$, and $t_{j}$ for all $j \in \omega$ such that $y_{j} \in M_{t_{j}}, t_{j} \leq s_{j}, \nu_{t_{j}}\left(e, y_{j}\right)=\nu, \nu_{s_{j}}\left(e, y_{j}\right)=\nu_{+},(\exists X)\left[\left(\nu_{+}, y_{j}\right) \in \mathcal{S}_{s_{j}}(X)\right]$, and $\nu_{+} \notin \mathcal{P}_{s_{j}}$.

For each $j$, let $v_{j}$ be least such that $y_{j} \in M_{v_{j}}$. Suppose that for some $j \in \omega$, $y_{j}$ had state $\nu$ at stage $v_{j}$. So $\tau=\emptyset$. Also, for any $\hat{y} \geq e$, if $\hat{v}$ is least such that $\hat{y} \in \hat{M}_{\hat{v}}$, then for some $\hat{\sigma} \subseteq\{0,1, \ldots, e\}, \hat{\nu}_{\hat{v}}(e, \hat{y})=(e, \hat{\sigma}, \emptyset) \| \nu$. Consequently, some $\hat{\nu} \| \nu$ occurs infinitely often in $\mathcal{S}(\hat{C})$.

So we may assume that no $y_{j}$ had state $\nu$ at stage $v_{j}$. For each $j$, let $u_{j}$ be least such that $\nu_{u_{j}}(e, y)=\nu_{t_{j}}(e, y)=\nu$. We may assume that there is some fixed $\nu_{-}$ such that for all $j, \nu_{u_{j}-1}(e, y)=\nu_{-}$. So $\nu_{-} \subset \nu$ and $\mathcal{H}_{\nu_{-}}$is infinite. Suppose that $\nu \geq \nu_{-}$. Since $\mathcal{H}_{\nu_{-}}$is infinite, by the induction hypothesis, some $\hat{\nu}$ with $\hat{\nu} \| \nu_{-}$(and therefore $\hat{\nu} \| \nu)$ occurs infinitely often in $\mathcal{S}(\hat{C})$. So assume $\nu \ngtr \nu_{-}$. So since $\nu_{-} \subset \nu$, $\nu_{-}=\left(e, \sigma, \tau_{-}\right)$for some $\tau_{-} \subset \tau$. Since for each $j$ we have $y_{j} \in \hat{V}_{i, u_{j}}-\hat{V}_{i, u_{j}-1}$ for some $i$, it is easy to see that either $y_{j} \in C_{2, u_{j}-1}$ or $y_{j} \in Q_{u_{j}} \cap Q_{u_{j}-1}$. We may assume that either $y_{j} \in C_{2, u_{j}-1}$ for all $j$ or $y_{j} \in Q_{u_{j}} \cap Q_{u_{j}-1}$ for all $j$.

Case 1. $y_{j} \in C_{2, u_{j}-1}$ for all $j$. By the assumption of the lemma and by the dual of Lemma 12, $\hat{\mathcal{H}}_{\hat{\nu}}$ is finite for each $\hat{\nu}$ with $|\hat{\nu}|<e$. Therefore for each $\hat{\nu}$ with $|\hat{\nu}|<e, \delta(\hat{\nu})$ is verified only finitely many times. So there are only finitely many occurrences in $\mathcal{I}$ of a state $\hat{\nu}$ with $|\hat{\nu}|<e$. For each $j$, let $\hat{\nu}_{j}$ be the state $\hat{\nu}$ which first occurs unmarked on $\left({ }^{+} \mathcal{I}_{u_{j}},{ }^{-} \mathcal{I}_{u_{j}-1}\right)$ such that $\hat{\nu} \| \nu_{u_{j}-1}\left(|\hat{\nu}|, y_{j}\right)$. Thus since for each $j, \hat{\nu}_{j}$ does not occur marked in $\mathcal{I}_{u_{j}-1}$ and is marked in $\mathcal{I}_{u_{j}}, \hat{\nu}_{j}$ has length $\geq e$ for all but finitely many $j$. Choose $\hat{\nu}$ of length $e$ such that $\hat{\nu}_{j}\lceil e=\hat{\nu}$ for infinitely many $j$. So by Lemma $11,(\exists X)[\hat{\nu}$ occurs infinitely often in $\mathcal{S}(\hat{X})]$. So by the dual of Lemma 8 , some $\hat{\nu}^{\prime} \geq \hat{\nu}$ occurs infinitely often in $\mathcal{S}(\hat{C})$. But by the definition of Rule $R_{3}$, Step $3, \hat{\nu} \| \nu$ (in fact, $\hat{\nu} \|_{*} \nu$ ), so $\hat{\nu}^{\prime} \| \nu$ as well.

Case 2. $y_{j} \in Q_{u_{j}} \cap Q_{u_{j}-1}$ for all $j$. Let $\hat{\nu}_{j}=\nu_{u_{j}}\left(d_{u_{j}-1}\left(y_{j}\right), y_{j}\right)^{*}$. Notice that $\hat{\nu}_{j}$ has length $d_{u_{j}-1}\left(y_{j}\right)$. We now show that (5) of Lemma 12 holds for $\nu_{0}=\nu_{-}$: Suppose that $\nu^{\prime} \subseteq \nu_{-}$and that $(\exists X)\left[\nu^{\prime}\right.$ occurs infinitely often in $\left.\mathcal{S}(X)\right]$. By Lemma 6(iii), either $\nu^{\prime} \in \mathcal{P}_{\omega}$ or $\mathcal{H}_{\nu^{\prime}}$ is infinite (setting $t=s$ in the definition of $\mathcal{H}_{\nu}$ ). If $\mathcal{H}_{\nu^{\prime}}$ is infinite, then by the induction hypothesis and the fact that $\nu^{\prime} \subseteq \nu_{-} \subset \nu$, some $\hat{\nu} \| \nu^{\prime}$ occurs infinitely often in $\mathcal{S}(\hat{C})$; the dual of Lemma 10 and Lemma 6(ii) then give $\nu^{\prime} \in \mathcal{P}_{\omega}$ after all.

So by Lemma 12, (6) of Lemma 12 also holds. So $d_{u_{j}-1}\left(y_{j}\right) \geq e$ for all but finitely many $j$. We may assume that there is some $\hat{\nu}$ of length $e$ such that $\hat{\nu}_{j} \succcurlyeq \hat{\nu}$ for all $j$. We may also assume either that $d_{u_{j}-1}\left(y_{j}\right)>e$ for all $j$ or that $d_{u_{j}-1}\left(y_{j}\right)=e$ for all $j$.

Case 2A. $d_{u_{j}-1}\left(y_{j}\right)>e$ for all $j$. Then $\hat{\nu}_{j} \succ \hat{\nu}$ for all $j$. But by the fact that $\mathcal{H}_{\nu}$ is infinite and by Lemma 12 , there is no state in $\mathcal{M}_{\omega}$ of length $>e$. But $\hat{\nu}_{j} \in \mathcal{M}_{u_{j}-1}$ 
for each $j$ by the definition of Rule $R_{4}$. So by Lemma $3, \hat{\nu}$ occurs in $\mathcal{S}(\hat{D})$ infinitely often. So by the dual of Lemma 8 , some $\hat{\nu}^{\prime} \geq \hat{\nu}$ occurs infinitely often in $\mathcal{S}(\hat{C})$. But for each $\hat{\nu}_{j}, \hat{\nu} \preceq \hat{\nu}_{j} \| \nu_{u_{j}}\left(\left|\hat{\nu}_{j}\right|, y_{j}\right) \succcurlyeq \nu$ (in fact $\left.\hat{\nu}_{j}=\nu_{u_{j}}\left(\left|\hat{\nu}_{j}\right|, y_{j}\right)^{*}\right)$. Thus $\hat{\nu} \| \nu$, and therefore $\hat{\nu}^{\prime} \| \nu$. So since $\hat{\nu}^{\prime}$ occurs infinitely often in $\mathcal{S}(\hat{C})$, we are done.

Case 2B. $d_{u_{j}-1}\left(y_{j}\right)=e$ for all $j$. So $\hat{\nu}_{j}=\hat{\nu}$ for all $j$. So $\hat{\nu}_{*} \| \nu_{-}$. By the induction hypothesis (which we can use since $\mathcal{H}_{\nu_{-}}$is infinite) and by the dual of Lemma 10, some $\hat{\nu}_{-}^{\prime} * \| \nu_{-}$occurs infinitely often in $\mathcal{S}(\hat{D})$. We may choose $\hat{\nu}_{-}^{\prime} * \| \nu_{-}$ such that no $\hat{\nu}_{-}^{\prime \prime}>_{*} \hat{\nu}_{-}^{\prime}$ occurs infinitely often in $\mathcal{S}(\hat{D})$. So $\hat{\nu}_{-}^{\prime} \in \mathcal{M}_{\omega}$ by Lemma 5 , by Lemma $12(2)$, and by the definition of $\mathcal{M}$. For each $\hat{\pi}_{*} \| \nu_{-}$, let $k_{\hat{\pi}}$ be the cardinality of $\left\{t<u_{j} \mid \zeta(\hat{\pi})\right.$ is verified at stage $\left.t\right\}$. By the definition of Rule $R_{4}$, $\hat{\nu}_{*} \| \nu_{-}$and for all $\hat{\pi}_{*} \| \nu_{-}, k_{\hat{\pi}} \leq k_{\hat{\nu}}$. But $\hat{\nu}_{-}^{\prime} * \| \nu_{-}$and $\left\{s \mid(\exists \hat{y})\left[\left(\hat{\nu}_{-}^{\prime}, \hat{y}\right) \in \mathcal{S}_{s}(\hat{D})\right]\right\}$ is infinite; therefore $\zeta\left(\hat{\nu}_{-}^{\prime}\right)$ is verified infinitely often. So $\zeta(\hat{\nu})$ is verified infinitely often, and therefore $\left\{s \mid(\exists \hat{y})\left[(\hat{\nu}, \hat{y}) \in \mathcal{S}_{s}(\hat{D})\right]\right\}$ is infinite. By the dual of Lemma 8 , some $\hat{\nu}^{\prime} \geq \hat{\nu}$ occurs infinitely often in $\mathcal{S}(\hat{C})$. Since $\hat{\nu} \| \nu$ (in fact $\hat{\nu}=\nu^{*}$ ), we are done.

Lemma 14. (1) $(\forall \nu)(\forall X)\left[\nu\right.$ occurs in $\mathcal{S}(X)$ infinitely often $\left.\Rightarrow \nu \in \mathcal{P}_{\omega}\right]$, (2) $(\forall \hat{\nu})(\forall \hat{X})\left[\hat{\nu}\right.$ occurs in $\mathcal{S}(\hat{X})$ infinitely often $\left.\Rightarrow \hat{\nu} \in \hat{\mathcal{P}}_{\omega}\right]$.

Proof. Prove (1) and (2) simultaneously by induction on the common length of $\nu$ and $\hat{\nu}$. Assume (1) and (2) hold for all $\nu$ such that $|\nu|<e$. We prove (1). The proof of (2) is analogous. Assume by way of contradiction that (1) fails for some $\nu$ of length $e$ and for some $X$. By Lemma 6 (iii) and by the definition of $\mathcal{H}_{\nu}, \mathcal{H}_{\nu}$ is infinite. By Lemma 13, some $\hat{\nu} \| \nu$ occurs infinitely often in $\mathcal{S}(\hat{C})$. By the dual of Lemma 10, some $\hat{\nu}^{\prime} * \|$ occurs infinitely often in $\mathcal{S}(\hat{D})$. Thus by Lemma 6(ii), $\nu \in \mathcal{P}_{\omega}$ after all.

Notice that for all $y, \lim _{s} d_{s}(y)$ exists, because $d_{s+1}(y) \leq d_{s}(y)$ for all $s$ such that $y \in M_{s}$. For all $y$, we put $d(y)=\lim _{s} d_{s}(y)$.

Lemma 15. (i) For every $e \in \omega$ there are only finitely many $y \in \omega$ such that $d(y)<e$.

(ii) If $y \in Q_{\omega}$ and $d(y) \geq 0$, then $\nu(d(y), y)^{*} \in \mathcal{M}_{\omega}$.

Proof. (i) By Lemma 14 and Lemma 12.

(ii) Fix $y$ and put $e=d(y)$. Choose $s_{0}$ so that for all $t \geq s_{0}$ we have $y \in Q_{t}$, $d_{t}(y)=e$, and $\nu_{t}(e, y)=\nu_{s_{0}}(e, y)$. If there were a $s \geq s_{0}$ such that $\nu_{s}(e, y)^{*} \notin$ $\mathcal{M}_{s}$, then Rule $R_{4}$ would make $\nu_{s+1}(e, y) \neq \nu_{s}(e, y)$, contradicting the fact that $\nu_{t}(e, y)=\nu_{s_{0}}(e, y)$ for all $t \geq s_{0}$. Thus for all $s, \nu(e, y)^{*}=\nu_{s}(e, y)^{*} \in \mathcal{M}_{s}$, and therefore $\nu(e, y)^{*} \in \mathcal{M}_{\omega}$.

Lemma 16. For every $\mu$, if there are infinitely many $y \in Q_{\omega}$ such that $\mu=$ $\nu(|\mu|, y)$, then there are infinitely many $\hat{y} \in \hat{P}_{\omega}$ such that $\mu^{*}=\hat{\nu}(|\mu|, \hat{y})$.

Proof. Assume that there are infinitely many $y \in Q_{\omega}$ such that $\mu=\nu(|\mu|, y)$. Let $\hat{\mu}=\mu^{*}$. We show $\hat{B}_{\hat{\mu}, \omega} \neq \emptyset$. (This suffices, since we can choose $\mu^{\prime} \succcurlyeq \mu$ of arbitrarily large length such that there are infinitely many $y \in Q_{\omega}$ such that $\mu^{\prime}=\nu\left(\left|\mu^{\prime}\right|, y\right)$.) Let $s_{0}$ be large enough so that $s_{0} \geq|\hat{\mu}|$ and $(\forall \pi \preceq \mu)\left(\forall s \geq s_{0}\right)\left[q_{s}(\pi)=q_{s_{0}}(\pi)\right]$ (cf. Lemma 2). Choose $y \in Q_{\omega}$ such that $\mu=\nu(|\mu|, y)$ and such that $d(y) \geq s_{0}$. Let $\hat{\pi}=\nu(d(y), y)^{*}$. So $\hat{\pi} \in \mathcal{M}_{\omega}$ by Lemma 15 , and thus for sufficiently large $t$, Condition (b) does not hold of $\hat{\pi}$ at stage $t$. So since $|\hat{\pi}|=d(y) \geq s_{0}$ and since 
$\hat{\mu} \preceq \hat{\pi}, \beta(\hat{\mu})$ must be verified only finitely often, and therefore $\left\{s \mid \hat{B}_{\hat{\mu}, s}=\emptyset\right\}$ must be finite. So $\hat{B}_{\hat{\mu}, \omega} \neq \emptyset$ by Lemma 7 .

Lemma 17. For every $\mu$, if there are infinitely many $y \in P_{\omega}$ such that $\mu=$ $\nu(|\mu|, y)$, then there are infinitely many $\hat{y} \in \hat{Q}_{\omega}$ such that $\mu^{*}=\hat{\nu}(|\mu|, \hat{y})$.

Proof. By Lemma $7, B_{\mu, \omega} \neq \emptyset$. So by Rule $R_{2}, \gamma\left(\mu^{*}\right)$ is verified only finitely often. Therefore $\left\{s \mid \hat{q}_{s}\left(\mu^{*}\right) \uparrow \vee(\exists t>s)\left[\hat{q}_{t}\left(\mu^{*}\right) \uparrow \vee \hat{q}_{t}\left(\mu^{*}\right) \neq \hat{q}_{s}\left(\mu^{*}\right)\right]\right\}$ is finite. So $\lim _{s} \hat{q}_{s}\left(\mu^{*}\right)$ exists. So (cf. the dual of Lemma 2) there is a $\hat{y} \in \hat{Q}_{\omega}$ such that $|\mu| \leq \hat{y}$ and $\mu^{*}=\hat{\nu}(|\mu|, \hat{y})$. To complete the proof of the lemma, notice that we can choose $\mu^{\prime} \succcurlyeq \mu$ of arbitrarily large length such that there are infinitely many $y \in P_{\omega}$ such that $\mu^{\prime}=\nu\left(\left|\hat{\mu}^{\prime}\right|, y\right)$.

Proof of Main Theorem. Lemmas 7, 16, and 17 and their duals show that for all states $\mu$, there are infinitely many $x$ such that $\mu=\nu(|\mu|, x)$ if and only if there are infinitely many $\hat{x}$ such that $\mu^{*}=\hat{\nu}(|\mu|, \hat{x})$. Also, the reader can easily verify by examining Case 3B of Section 4 that $U_{i}={ }^{*} W_{i}^{A}$ and $V_{i}={ }^{*} W_{i}^{B}$. Finally, as observed in Section 5, there are recursive functions $g, h: \omega \rightarrow \omega$ such that $\hat{V}_{i}={ }^{*} W_{g(i)}^{A}$ and $\hat{U}_{i}={ }^{*} W_{h(i)}^{B}$ for all $i \in \omega$. Thus (1)-(3) of Section 3 hold, so by (4) of Section 3, $\left(\mathcal{E}^{A}\right)^{*}$ and $\left(\mathcal{E}^{B}\right)^{*}$ are effectively isomorphic.

\section{REFERENCES}

1. L. Feiner, Hierarchies of Boolean algebras, J. Symbolic Logic 35 (1970), 365-374. MR 44:39

2. T Hammond, Nonisomorphism of lattices of recursively enumerable sets, J. Symbolic Logic $\mathbf{5 8}$ (1993), 1177-1188. MR 95c:03100

3. A. H. Lachlan, On the lattice of recursively enumerable sets, Trans. Amer. Math. Soc. 130 (1968), 1-37. MR 37:2594

4. W. Maass, Characterization of recursively enumerable sets with supersets effectively isomorphic to all recursively enumerable sets, Trans. Amer. Math. Soc. 279 (1983), 311-336. MR 85e:03099

5. H. Rogers, Jr., Theory of Recursive Functions and Effective Computability, McGraw-Hill, New York, 1967. MR 37:61

6. R. M. Smullyan, Theory of formal systems, Annals of Mathematics Studies, no. 47, Princeton, N. J., 1961. MR 22:12042; MR 27:2409

7. R. I. Soare, Automorphisms of the lattice of recursively enumerable sets, Part I: Maximal sets, Ann. of Math. (2) 100 (1974), 80-120. MR 50:12685

8. R. I. Soare, Automorphisms of the lattice of recursively enumerable sets, Part II: Low sets, Ann. Math. Logic 22 (1982), 69-107. MR 83k:03048

9. R. I. Soare, Recursively enumerable sets and degrees: a study of computable functions and computably generated sets, Springer-Verlag, Berlin, Heidelberg, New York, London, Paris, Tokyo, 1987. MR 88m:03003

Division of Mathematics and Computer Science, Truman State University, Kirksville, Missouri 63501

E-mail address: thammond@math.nemostate.edu 